\title{
Extrapolated Shock Tracking: bridging shock-fitting and embedded boundary methods
}

\author{
Mirco Ciallella ${ }^{\mathrm{a}}$, Mario Ricchiuto ${ }^{\mathrm{a}}$, Renato Paciorri $^{\mathrm{b}}$, Aldo Bonfigliolic \\ ${ }^{a}$ Team CARDAMOM, INRIA Bordeaux Sud-Ouest, 33405 Talence, France \\ ${ }^{b}$ Dip. di Ingegneria Meccanica e Aerospaziale, Università di Roma "La Sapienza", Via \\ Eudossiana 18, 00184 Rome, Italy \\ ${ }^{c}$ Scuola di Ingeneria - Università degli Studi della Basilicata, Viale dell'Ateneo Lucano \\ 10, 85100 Potenza, Italy
}

\begin{abstract}
We propose a novel approach to approximate numerically shock waves. The method combines the unstructured shock-fitting approach developed in the last decade by some of the authors, with ideas coming from embedded boundary techniques. The numerical method obtained allows avoiding the remeshing phase required by the unstructured fitting method, while guaranteeing accuracy properties very close to those of the fitting approach. This new method has many similarities with front tracking approaches, and paves the way to shock-tracking techniques truly independent on the data and mesh structure used by the flow solver. The approach is tested on several problems showing accuracy properties very close to those of more expensive fitting methods, with a considerable gain in flexibility and generality.
\end{abstract}

Keywords: Shock-fitting, unstructured-grids, embedded-boundary

\section{Introduction}

The numerical techniques used to simulate flows with shock-waves are essentially two: the widely used shock-capturing (SC) methods, and the less common shock-fitting (SF) methods. The former relies on the proven mathematical legitimacy of weak solutions: all types of flows, including flows with shocks, can be computed by using the same discretization of the equations in divergence form. Nevertheless, the shocks always appear smeared in a region whose thickness is of two or three cells rather than actual discontinuities. In addition to this, but perhaps more correctly because of this, since the states 
of the cells inside this region are unphysical [1], the shock-capturing methods suffer from some numerical problems concerning the stability, the accuracy and the quality of the solutions that sometimes give anomalous results. A catalogue of these failings was made by Quirk in the early 90s of the last century [2]. Despite the great efforts made by numerous researchers in the last decades to develop shock-capturing methods, these numerical problems are not entirely solved and still plague the numerical solutions obtained by shock-capturing solvers.

The shock-fitting technique for compressible flow computations has been developed by Gino Moretti [3, 4] in the 1960s. It consists in explicitly identifying the shock as a line (surface in 3D) within the flow-field and computing its motion and upstream and downstream states according to the Rankine-Hugoniot equations. However, historically, the techniques developed by Moretti and his collaborators were designed for solvers based on structured grids and this made their development very difficult and complex, especially when extended to flows with shock interactions [4].

Two different shock-fitting methodologies blossomed between the 60s and 80s: the boundary shock-fitting and floating shock-fitting. In the former approach, the shock is made to coincide with one of the boundaries of the computational domain so that the treatment of the jump relations across the shock is confined to the boundary points. Even though this method greatly simplified the coding, the treatment of shocks appearing within the computational domain and of shock interactions became a major challenge. The floating shock-fitting approach was developed to be capable of dealing with more complex flow configurations. In the floating version, discontinuities can freely move over a background structured mesh: a shock front is described by its intersections with the grid-lines, which give rise to $x$ and $y$ shock points, meaning that they are allowed to move onto grid-lines. Even though floating shock-fitting codes have been used with success in the past to compute steady and un-steady two- and three-dimensional flows involving shock reflections and shock interactions $[5,6,7]$, they are very complex to code and require extensive changes in the computational kernel of the gas-dynamic solver.

In the 80 s and early 90 s the CFD community has shown increasing interest in unstructured meshes. This is mainly due to the features that characterize this kind of grids: the ability to easily mesh complex geometries and the possibility of locally adapting the mesh size to follow the flow features. The latter advantage makes them well-suited to simulate compressible flows with shock waves and contact discontinuities. Exploiting this flexibility, Pa- 
ciorri and Bonfiglioli developed a new unstructured shock-fitting technique for unstructured vertex-centered solvers, described in [8]. This approach has alleviated many of the difficulties of the shock-fitting techniques in the structured-grid framework. In recent years, the unstructured shock-fitting technique was improved to deal with interactions among discontinuities in two-dimensional flows [9], three-dimensional flows [10] and un-steady compressible flows $[11,12]$ opening a new route in simulating flow-field with shock waves. In particular, not only shocks and contact discontinuities are fitted, but also the interaction points, for example the triple points arising in Mach reflections [13]. A limitation of this technique is that it heavily relies on the flexibility of triangular and tetrahedral grids to locally produce a fitted unstructured grid around the discontinuities. This limits its application to unstructured vertex-centered codes.

Recently, the research group headed by Prof. J. Liu proposed and developed a shock-fitting technique for unstructured cell-centered solvers [14, 15]. However, even this technique has an important limitation: it uses a deforming grid whose topology cannot be changed during the computation, unless an expensive re-meshing (and, consequently, interpolation of the solution) is carried out. This is an important restriction, especially when shock-waves move throughout the flow-field or whenever new shocks appear during the computation.

Both these shock-fitting formulations currently available heavily rely on the data structure of the flow solver, and more particularly on the mesh. Indeed, in all these techniques, the jump conditions are attached to some mesh entity (edge, face, or node). This very often makes the methods better suited for one or another family of flow solvers (node-centered, cell-centered, finite volume, finite element etc.), thus limiting its use. Another complication is that both these methods require the mesh to follow exactly the evolution of the shock wave, which puts additional requirements on the meshing/remeshing techniques used.

In this work we aim at proposing a new approach, which is in some way more general and flexible. The initial idea comes from the similarity between the constraints arising from shock-fitting, and those related to the construction of boundary-fitted grids for simulating flows around complex geometries. In this context, immersed and embedded boundary methods have been developed since many years to allow a flexible management of complex geometries. The two approaches rely on a slightly different philosophy.

Immersed methods are based on an extension of the flow equations out- 
side the physical domain (typically within solid bodies). This extension is formulated using some smooth approximation of the Dirac delta function to localize the boundary, as well as to impose the boundary condition. These methods are relatively old, and based on the original ideas of Peskin [16]. Finite element and unstructured mesh extensions for elliptic PDEs as well as for incompressible, and compressible flows have been discussed in $[17,18,19]$.

Embedded methods, on the other hand, solve the PDEs only in the physical domain, while replacing the exact boundary with some more or less accurate approximation, combined with some weak enforcement of the boundary condition. There is a certain number of techniques to perform this task, which go from the combination of XFEM-type methods with penalization or Nitsche's type approaches [20], to several types of cut finite element methods with improved stability [21, 22], to approximate domain methods such as the well known ghost-fluid method [23, 24], and the more recent shifted boundary method (SBM) [25, 26].

In this work we borrow ideas from approximate domain methods, and in particular from the SBM. As in the latter, we impose modified conditions on surrogate shock-manifolds, acting as boundaries between the shock-upstream and shock-downstream regions. These surrogate boundaries are composed of two sets of mesh faces enclosing the cavity of elements crossed by the shock. The values of the flow variables imposed on these surrogate boundaries are extrapolated from the tracked shock front accounting for the non-linear jump and wave propagation conditions, as done in the unstructured shock-fitting approach. As in the SBM, the extrapolation is based on a truncated Taylor series expansion from the surrogate boundaries to the front, allowing to preserve the overall accuracy of the discretization. This paper, in particular, only deals with second-order piecewise linear approximations, but all the ideas can be extended to higher order. Note however that differently from e.g. the extension of the SBM to hyperbolic problems [27], the approach proposed here requires the solution of three coupled problems: the CFD upstream of the shock, the CFD downstream of the shock, the coupled algebraic system obtained from the Rankine-Hugoniot relations augmented with the characteristic information traveling toward the shock front. As in shock-fitting and front tracking methods [28], the shock front is explicitly discretized by an independent lower-dimensional mesh, and its position, as well as the position of the two surrogate boundaries, are themselves part of the computational result. These elements make the present work not only original w.r.t. previous shock-fitting methods, but also with respect to previous work in embedded 
methods and in particular the SBM approach. Indeed, the most recent work on the use of similar ideas, only considers interfaces independent on the solution, and linear elliptic partial differential equations [29]. Moreover, the approach proposed in the reference is based on a single surrogate interface, while the approach proposed here uses a symmetric formulation with two surrogates. The resulting method bears some similarities to front tracking approaches, and for this reason is referred to as extrapolated Shock Tracking (eST) to differentiate it from previous unstructured shock-fitting methods in which the faces of the shock mesh are part of the CFD meshes. This new method constitutes a bridge between shock-fitting and embedded boundary methods. It removes some of the constraints of the approach by Paciorri and Bonfiglioli, while keeping its flexibility. The method proposed is actually even more general as it constitutes a shock-fitting/tracking technique virtually independent on the data structure of the underlying gas-dynamic solver. This paper focuses on the formulation of the method in two-space dimensions, and on its validation on classical problems involving strong shocks, as well as on problems with shock interactions, where a capability for hybrid fitting-capturing computations is shown.

\section{Generalities}

We consider the numerical approximation of solutions of the steady limit of the Euler equations reading:

$$
\partial_{t} \mathbf{U}+\nabla \cdot \mathbf{F}=0 \quad \text { in } \quad \Omega \subset \mathbb{R}^{d}
$$

with conserved variables and fluxes given by:

$$
\mathbf{U}=\left[\begin{array}{c}
\rho \\
\rho \mathbf{u} \\
\rho E
\end{array}\right], \quad \mathbf{F}=\left[\begin{array}{c}
\rho \mathbf{u} \\
\rho \mathbf{u} \otimes \mathbf{u}+p \mathbb{I} \\
\rho H \mathbf{u}
\end{array}\right]
$$

having denoted by $\rho$ the mass density, by $\mathbf{u}$ the velocity, by $p$ the pressure, and with $E=e+\mathbf{u} \cdot \mathbf{u} / 2$ the specific total energy, $e$ being the specific internal energy. Finally, the total specific enthalpy is $H=h+\mathbf{u} \cdot \mathbf{u} / 2$, with $h=e+p / \rho$ the specific enthalpy. For simplicity in this paper we work with the classical perfect gas equation of state:

$$
p=(\gamma-1) \rho e
$$


with $\gamma$ the constant (for a perfect gas) ratio of specific heats. However, note that the method discussed allows in principle to handle any other type of gas, see e.g. [30].

In all applications involving high-speed flows, solutions of (1) are only piecewise continuous. In $d$ space dimensions, discontinuities are represented by $d-1$ manifolds governed by the well known Rankine-Hugoniot jump conditions reading:

$$
\llbracket \mathbf{F} \cdot \mathbf{n} \rrbracket=w \llbracket \mathbf{U} \rrbracket
$$

having denoted by $\mathbf{n}$ the local normal vector to the shock, by $\llbracket \cdot \rrbracket$ the corresponding jump of a quantity across the discontinuity, and with $w$ the normal component of the shock speed.

As discussed in the introduction, the method proposed exploits ideas from two different approaches: the unstructured shock fitting method [8] and subsequent works; the shifted boundary method by [25] and subsequent works. In the following sections we recall the main ingredients of these two techniques.

\section{Unstructured shock-fitting algorithm}

We shall first briefly describe the unstructured shock-fitting technique developed by Paciorri and Bonfiglioli $[8,9,10]$, in the following referred to with the acronym SF.

In this approach the set of dependent variables is available within all gridpoints of a tessellation (made of triangles in 2D and tetrahedra in 3D) that covers the entire computational domain; this is what we call the background mesh. In addition to the background mesh, the fitted discontinuities (either shocks or slip-streams) are discretised using a collection of points which are mutually joined to form a connected series of line segments, as shown in Fig. 1a for the 2D case, or a triangulated surface in 3D, as shown in Fig. 1c. This is what we call the shock mesh. For example, the thick solid (yellow) line in Fig. 1a marks the various fitted discontinuities that arise due to the interaction between two shocks of the same family: the two incident shocks, the resulting shock, a weak compression wave ${ }^{1}$ and the slip-stream located between the former two. Figure 1c, which refers to the three-dimensional, supersonic flow past a blunt-nosed object, shows the triangulated surfaces used

\footnotetext{
${ }^{1}$ could be an expansion wave instead, depending on the upstream boundary conditions
} 


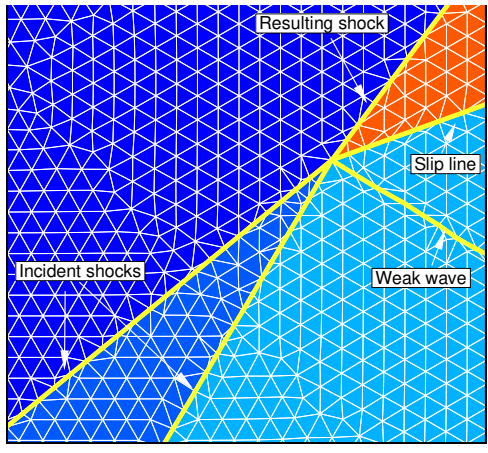

(a) Interaction of two shocks of the same family

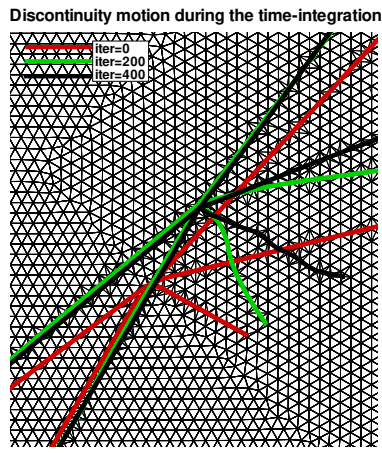

(b) Pseudo-temporal evolution of the grid and the fitted discontinuities

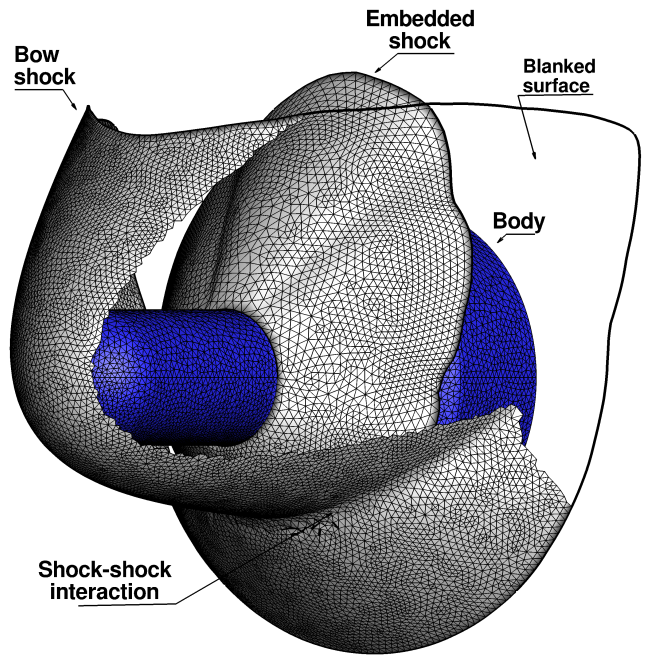

(c) Supersonic flow over a blunt-nosed body.

Figure 1: Examples of fitted discontinuities on unstructured meshes.

to fit the bow shock and the imbedded shock that arises at the cylinder-flare junction.

Although it is not evident from Fig. 1, each fitted discontinuity is a doublesided internal boundary of zero thickness. Being the width of the discontinuity negligible, its two sides are discretised using the same polygonal curve 
or triangulated surface, so that each pair of nodes that face each other on the two sides of the discontinuity share the same geometrical location, but store different values of the dependent variables, one corresponding to the upstream state and the other to the downstream one. Moreover, a velocity vector normal to the discontinuity is assigned to each pair of grid-points on the fitted discontinuity: it represents the displacement velocity of the discontinuity. The initial condition for a shock-fitting calculation is typically (see [15] for a different approach) supplied by running a shock-capturing calculation on the background mesh; then, a feature extraction algorithm, such as the those described in $[31,15,32]$, is used to provide the initial (though approximate) location of the discontinuities. Even when dealing with steady flows, the approach is inherently time-dependent, because both the solution and the grid change with time, due to the displacement of the fitted discontinuities. Whenever a steady solution exists, the shock speed asymptotically vanishes and the tessellation of the flow domain does not any longer change. This is illustrated in Fig. 1b which shows the pseudo-temporal evolution of the various discontinuities involved in the shock-interaction of Fig. 1a. Moreover, Fig. 1b reveals that the spatial location of the fitted discontinuities is independent of the location of the grid-points that make up the background grid and that local re-meshing only takes place in the immediate neighborhood of the moving discontinuities.

\section{Shifted-boundary method}

The main advantage of embedded boundary methods, and among them the SBM $[25,26]$, is the ease of mesh generation with respect to the classical body-fitted methods. It has been pointed out how trivial this task might be even when complicated geometries are taken into account. With all the benefits that characterize these approaches, some shortcomings arose in the standpoint of the enforcement of boundary conditions. The originality of the SBM lies in the idea of shifting the location where the boundary conditions are applied. In order to guarantee consistency, and retain the mesh convergence rates of the original method, the boundary conditions have to be modified.

The main steps of the method are the following. Given a mesh including the physical domain $\Omega$, not conformal w.r.t. the domain boundary $\Gamma$, one must first define a surrogate boundary $\tilde{\Gamma}$. As shown in figure $2 \mathrm{a}, \tilde{\Gamma}$ is essentially built from the mesh faces and mesh points in $\Omega$ closest to the true boundary 


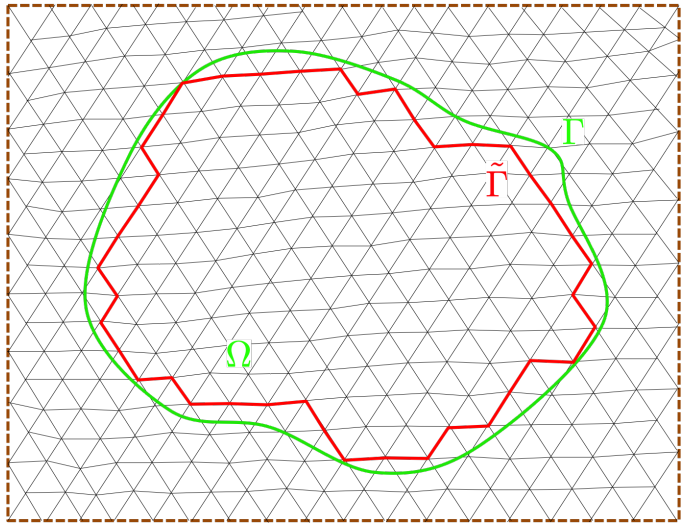

(a) The surrogate boundary $\tilde{\Gamma}$ and true boundary $\Gamma$

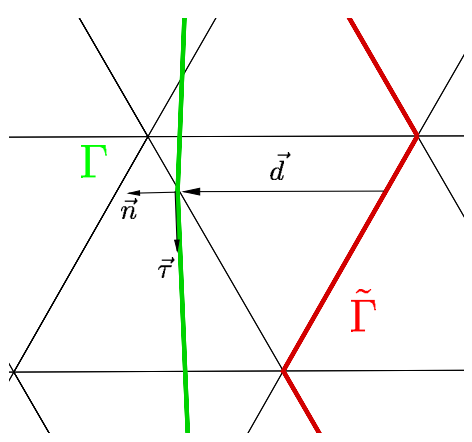

(b) The distance vector $\vec{d}$ and unit normal and tangent vectors to the true boundary

Figure 2: The SBM: the surrogate and actual boundaries, and the distance vector $\vec{d}$.

$\Gamma$. Next, for any point of the surrogate boundary $\tilde{\Gamma}$, one needs to be able to define a map to a unique point of the true boundary $\Gamma$ :

$$
\begin{gathered}
\mathbf{M}: \tilde{\Gamma} \rightarrow \Gamma \\
\tilde{\mathbf{x}} \rightarrow \mathbf{x}
\end{gathered}
$$

which maps $\tilde{\mathbf{x}} \in \tilde{\Gamma}$ on the surrogate boundary to $\mathbf{x} \in \Gamma$ on the true boundary. The map $\mathbf{M}$ can be built in several ways, for example using a closest point projection, or using level sets, or equivalently using distances along directions normals to the true boundary $\Gamma$, as shown in Fig. 2a. Since the gap between $\tilde{\Gamma}$ and $\Gamma$ is going to be of crucial importance, in terms of accuracy of the solution, the map $\mathbf{M}$ will be characterized through a distance vector function:

$$
\mathbf{d}_{\mathbf{M}}(\tilde{\mathbf{x}})=\mathbf{x}-\tilde{\mathbf{x}}=[\mathbf{M}-\mathbf{I}](\tilde{\mathbf{x}})
$$

If $\mathbf{M}$ is built using distances along normals to $\Gamma$, the vector $\mathbf{d}_{\mathbf{M}}(\tilde{\mathbf{x}})$ is parallel to the normal to $\Gamma$ in $\tilde{\mathbf{x}}$. Finally, the boundary conditions have to be modified to provide high-order (at least second-order) convergence rate of the solution. This can be accomplished by writing a Taylor expansion formula 
centered at $\tilde{\mathbf{x}} \in \tilde{\Gamma}$, recalling Eq. (7):

$$
\begin{aligned}
u(\mathbf{x}) & =u(\tilde{\mathbf{x}})+\nabla u(\tilde{\mathbf{x}}) \cdot(\mathbf{x}-\tilde{\mathbf{x}})+O\left(\|\mathbf{x}-\tilde{\mathbf{x}}\|^{2}\right) \\
& =u(\tilde{\mathbf{x}})+\nabla u(\tilde{\mathbf{x}}) \cdot(\mathbf{M}(\tilde{\mathbf{x}})-\tilde{\mathbf{x}})+O\left(\|\mathbf{M}(\tilde{\mathbf{x}})-\tilde{\mathbf{x}}\|^{2}\right) \\
& =u(\tilde{\mathbf{x}})+\nabla u(\tilde{\mathbf{x}}) \cdot \mathbf{d}_{\mathbf{M}}(\tilde{\mathbf{x}})+O\left(\left\|\mathbf{d}_{\mathbf{M}}(\tilde{\mathbf{x}})\right\|^{2}\right)
\end{aligned}
$$

Equation (8) is at most second-order accurate, unless additional terms in the Taylor expansion are included, as explained in [33]. Now, if on $\Gamma$ the prescribed boundary condition is $u(\mathbf{x})=g(\mathbf{x})$, the main idea of the SBM is

to deduce from Eq. (8) that the boundary condition to be imposed on $\tilde{\Gamma}$ to allow for second order of accuracy w.r.t. $\left\|\mathbf{d}_{\mathbf{M}}(\tilde{\mathbf{x}})\right\|$ is

$$
u(\tilde{\mathbf{x}})=g(\mathbf{M}(\tilde{\mathbf{x}}))-\nabla u(\tilde{\mathbf{x}}) \cdot \mathbf{d}_{\mathbf{M}}(\tilde{\mathbf{x}}) .
$$

This extrapolation constitutes the main idea exploited in the following.

\section{Extrapolated Shock-Tracking}

We discuss here the extrapolated Shock-Tracking (eST) method we propose. We focus on steady state flows in at most two space dimensions, however most of the ideas discussed can be generalized to three space dimensions. The eST algorithm can be summarized in three main steps allowing to update the computational domains and solution values that leads from the available mesh and solution at pseudo-time $t$ to an updated mesh and solution at pseudo-time $t+\Delta t$ :

1. (Shock/background-mesh coupling) Geometrical coupling of the shockmesh with the background-mesh, and definition of separate shockupstream and shock-downstream computational domains;

2. (Computational domain update) Iteration evolving in (pseudo-)time the flow variables in each computational domain independently;

3. (Shock update) Evolution of the position of the shock and of the flow variables values at the shock, using the jump relations (4).

These three steps, are applied iteratively until a steady state is obtained, or, when dealing with un-steady flows, in a time-accurate manner $[11,12]$. 
The most specific ingredients of the method are those of steps 1 and 3 . Indeed, step 2 essentially relies on the use of an accurate multidimensional upwind unstructured grid solver to compute the smooth flows upstream and downstream of the shock, and additional discontinuities not being fitted by the above method. We will briefly recall in Sect. 5.4 the cell-vertex solution method used here.

The main difference between the technique described here and the one proposed by Paciorri and Bonfiglioli $[8,9,10]$ is in step 1 . Indeed, the present technique removes the need to insert the shock-mesh in the background mesh, which can be a critical aspect, especially when different shock-surfaces mutually interact in the three dimensional space [10]. To this end, we exploit ideas coming from embedded boundary methods. In particular, we propose to use an extrapolation from the background mesh to the shock mesh in the spirit of the SBM initially proposed in [25] for elliptic problems and extended to hyperbolic problems in [27]. In other words, the method proposed consists in replacing re-meshing with the definition of sufficiently accurate extrapolation functions, which allow the transfer of information between the background and shock meshes. This allows to completely remove the need of re-meshing.

As already mentioned in the introduction, we refer to this new method as to extrapolated Shock-Tracking (eST) to differentiate it from unstructured shock fitting, in which a conformal mesh fitting the shock front is generated, and to differentiate it from the SBM, in which the true boundary is replaced by a unique surrogate with extrapolated boundary values. The eST method actually has similarities with high-order front tracking approaches [28], and also for this reason we prefer referring to it as shock-tracking. It may also be viewed as some sort of elaborate solution optimization procedure in which, starting from a captured result, one iteratively places the shock front and modifies the flow solution by solving the nonlinear jump conditions. In this respect there are similarities with approaches based on jump minimization coupled with mesh adaptation as those recently proposed e.g. by $[34,35]$ in the framework of Discontinuous Galerkin methods. The details of the method are discussed in the next sections, highlighting the major changes and differences w.r.t. the SF approach of $[8,9,10]$.

\subsection{Geometrical setting}

To illustrate the algorithmic features of the eST method, let us consider a two-dimensional domain and a shock front crossing the domain at a given 
time $t$ (see Fig. 3a). The shock front is described by a collection of shockedges whose endpoints are the shock-points, marked by squares in Fig. 3a. Shock-edges and shock-points make up the shock-mesh. A background triangular mesh, whose grid-points are denoted by circles in Fig. 3a, covers the entire computational domain. It is noted that the position of the shock-points is completely independent of the location of the grid-points of the background mesh. While each grid-point of the background mesh is characterized by a single set of dependent variables, two sets of values, corresponding to the upstream and downstream states, are assigned to each shock-point. We assume that at time $t$ the solution is known at all grid- and shock-points. The computation of the subsequent time level $t+\Delta t$ can be split into several steps that will be described in detail in the following sub-sections.

\subsection{Cell removal around the shock front}

The first step consists in the removal of the triangles crossed by the shock, see Fig. 3b. By doing so, a hole is dug within the background mesh that, contrary to the technique proposed in [8], is not re-meshed. The creation of the hole splits the background mesh into two disjoint sub-domains which do not include the shock. Instead, we label certain boundaries as "surrogate" shock-boundaries which will be used to couple the flow domains, via the shock relations. We shall hereafter call "computational mesh" the background mesh with the triangles within the hole being removed. It is worth noting that the number of grid-points of the computational mesh is the same as that of the background grid, whereas the number of triangular cells is less, due to the cell removal. Hereafter, the upstream and downstream surrogate boundaries, drawn using red lines in Fig. $3 \mathrm{~b}$, will be called $\tilde{\Gamma}_{U}$ and $\tilde{\Gamma}_{D}$. Furthermore, the shock-boundary, which represents the actual shock position, will be referred to as $\Gamma$ and its upstream and downstream sides as $\Gamma_{U}$ and $\Gamma_{D}$, respectively. Finally, a second surrogate boundary located within the shock-downstream

sub-domain (the blue line in Fig. $3 \mathrm{~b}$ ) will be called $\hat{\Gamma}_{D}$. This second surrogate boundary is obtained by removing all cells that have one, or more, nodes on $\tilde{\Gamma}_{D}$.

\subsection{Computation of the tangent and normal unit vectors}

In order to apply the Rankine-Hugoniot jump relations, Eq. (4), the tangent and normal unit vectors along the shock-front have to be calculated within each pair of shock-points. The tangent unit vector $\boldsymbol{\tau}_{i}$ in shock-point 


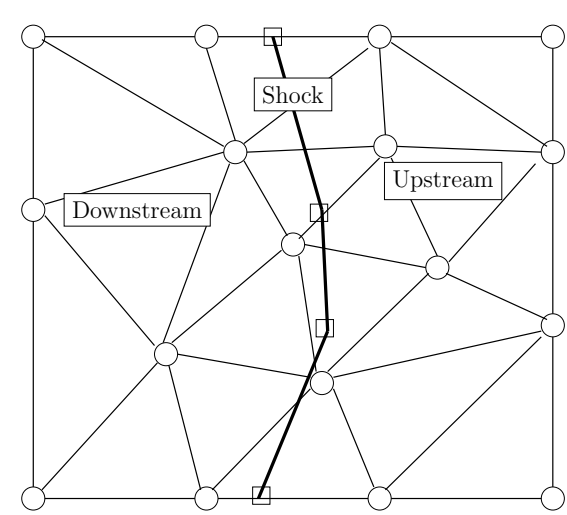

(a) Shock-mesh laid on top of the background-mesh

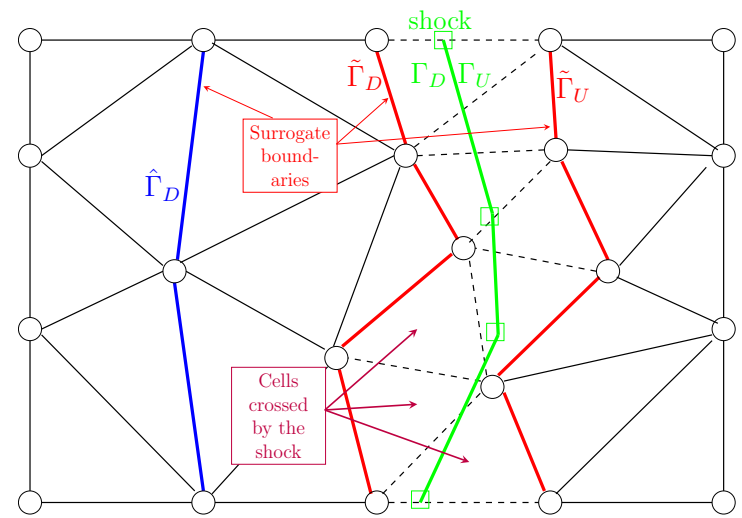

(b) Shock-mesh, computational-mesh and surrogate boundaries

Figure 3: The computational-mesh is obtained by removing those cells of the background mesh that are crossed by the shock-mesh.

$i$ is obtained from:

$$
\boldsymbol{\tau}_{i}=\frac{\mathbf{v}_{\tau_{i}}}{\left|\mathbf{v}_{\tau_{i}}\right|}
$$

where $\mathbf{v}_{\tau_{i}}$ is the vector tangent to the shock-front in shock-point $i$. The normal unit vector $\mathbf{n}_{i}$ is perpendicular to $\tau_{i}$ and such that it points from the shock-downstream towards the shock-upstream region. The computation of $\mathbf{v}_{\tau_{i}}$ relies on finite difference formulae which involve the coordinates of the shock-point itself and those of its neighboring shock-points. By reference to Fig. 4, $\mathrm{x}\left(P_{i}^{t}\right)$ denotes the position of shock-point $i$ at time level $t$. Shockpoints $i-1$ and $i+1$ are located on both sides of shock-point $i$ and their position $\mathrm{x}\left(P_{i-1}^{t}\right)$ and $\mathrm{x}\left(P_{i+1}^{t}\right)$ at time level $t$ can be used to compute the tangent and normal unit vectors in shock-point $i$. A preliminary test is required to verify whether these adjacent shock-points belong to the domain of dependence of shock-point $i$. This is easily checked using the following inequality:

$$
\mathbf{u}_{d, i+1}^{t} \cdot \boldsymbol{\tau}_{i+\frac{1}{2}}-a_{d, i+1}^{t}<0
$$

where:

$$
\boldsymbol{\tau}_{i+\frac{1}{2}}=\frac{\mathbf{x}\left(P_{i+1}^{t}\right)-\mathbf{x}\left(P_{i}^{t}\right)}{l_{i+\frac{1}{2}}} \quad l_{i+\frac{i}{2}}=\left|\mathbf{x}\left(P_{i+1}^{t}\right)-\mathbf{x}\left(P_{i}^{t}\right)\right|
$$




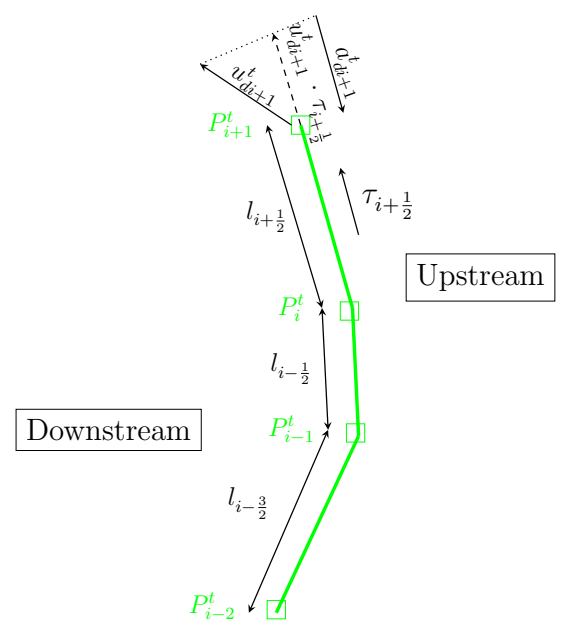

Figure 4: Test needed to check whether point $P_{i+1}$ belongs to the domain of dependence of $P_{i}$.

and $\mathbf{u}_{d, i+1}^{t}$ and $a_{d, i+1}^{t}$ are the shock-downstream flow and acoustic velocity in shock-point $i+1$ at time level $t$. If Eq. (10) is verified, shock-point $i+1$ falls within the domain of dependence of shock-point $i$. Once this test has been repeated in shock-point $i-1$, three different situations may arise:

1. both shock-points $i-1$ and $i+1$ are in the domain of dependence of shock-point $i$;

2. only shock-point $i-1$ is in the domain of dependence of shock-point $i$;

3. only shock-point $i+1$ is in the domain of dependence of shock-point $i$;

When case 1 applies, the computation of $\mathbf{v}_{\tau_{i}}$ must involve the shock-points on both sides; therefore:

$$
\mathbf{v}_{\tau_{i}}=\tau_{i+\frac{1}{2}} l_{i-\frac{1}{2}}^{2}+\tau_{i-\frac{1}{2}} l_{i+\frac{1}{2}}^{2}
$$

When case 2 applies, shock-point $i+1$ must not be used in the computation of the tangent vector $\mathbf{v}$, and the following upwind-biased formula, which involves shock-point $i-2$, instead of $i+1$, is used:

$$
\mathbf{v}_{\tau_{i}}=\tau_{i-\frac{1}{2}}\left(l_{i-\frac{1}{2}}+l_{i-\frac{3}{2}}\right)^{2}+\left(\tau_{i-\frac{1}{2}}+\tau_{i-\frac{3}{2}}\right) l_{i-\frac{1}{2}}^{2}
$$

Finally, the third case is specular to the second one, but the corresponding formula involves shock-points $i, i+1$ and $i+2$. 
The finite difference approximations (12) and (13) are both second-orderaccurate even if the shock-points are un-evenly spaced along the shock-front.

\subsection{Solution update using the CFD solver}

The solution is updated to time level $t+\Delta t$ using an unstructured shockcapturing code. The flow solver uses the computational mesh built in step 5.2, which includes the surrogate shocks $\tilde{\Gamma}_{U}$ and $\tilde{\Gamma}_{D}$ as part of its boundary. In particular, the flow computations are performed on two non-communicating domains separated by the hole bounded by the surrogate shock-boundaries (see Fig. 5). As already mentioned, each shock-point consists in two superimposed points of the shock-mesh: one of these represents the shockdownstream state and the other the shock-upstream one. Even though these points are not part of the flow domain, they will play a central role in the coupling of the surrogate shock-boundaries, as we will see in the next sections.

Concerning the solver used in this paper, it is based on a Residual Distribution $(R D)$ method evolving in time approximation of the values of the flow variables in mesh nodes. The method has several appealing characteristics, including the possibility of defining genuine multidimensional upwind strategies for Euler flows, by means of a wave decoupling exploiting appropriately preconditioned forms of the equations [36]. By combining ideas from both the stabilized finite element and finite volume methods, these schemes allow to achieve second order of accuracy and monotonicity preservation with a compact stencil of nearest neighbors. The interested reader can refer to $[37,38]$ and references therein for an in-depth review of this family of methods, as well as to $[36,39]$ and references therein for some specific choices of the implementation used here.

Note that the choice of the flow solver is somewhat independent on the rest of the method object of this paper. Concerning the presentation in the following sections, the main impact of our choice is on the structure of the solver which is assumed to be evolving nodal values of the unknowns. Cell based discretization methods can be easily accommodated by minor modifications of the transfer operators discussed later in the paper.

\subsection{Solution transfer from/to the shock to/from the surrogates}

The flow solver provides updated nodal values within all grid-points of the computational mesh at time level $t+\Delta t$. The shock-upstream surrogate 


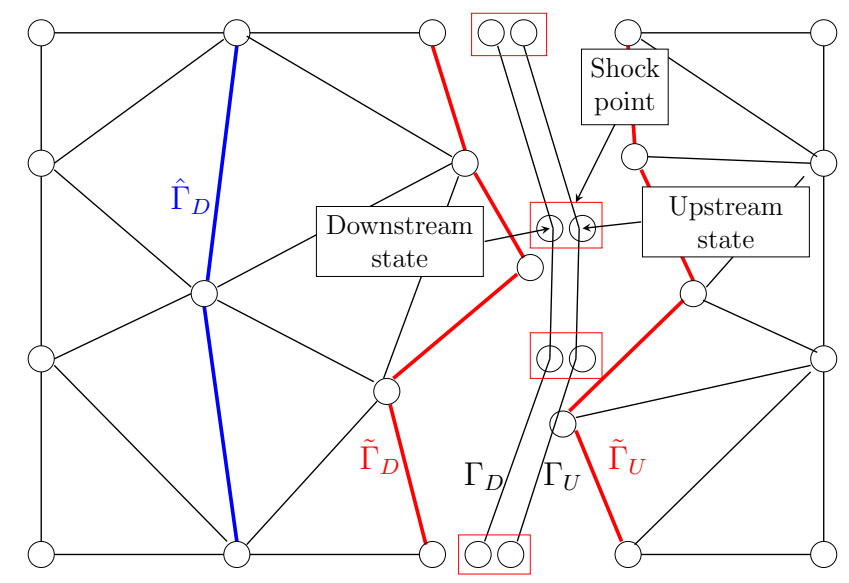

Figure 5: The solution update is performed using the computational mesh.

boundary, $\tilde{\Gamma}_{U}$ behaves like a supersonic outflow and, therefore, no boundary conditions should be applied. The situation along the shock-downstream surrogate $\tilde{\Gamma}_{D}$ is however different, since the flow is subsonic in the shocknormal direction and, therefore, boundary conditions corresponding to the downstream-running waves (the 'fast' acoustic, entropy and vorticity waves) are missing. Moreover, the upstream and downstream states of the shockpoints have not been updated, since the shock-mesh is not part of the computational mesh. To perform this update, one needs to define appropriate transfer operators from the surrogate shock-boundaries to the shock. Due to the use of an upwind discretization in the CFD solver, we assume that the only variable that has been correctly computed along the shock-downstream surrogate boundary is the Riemann variable associated with the acoustic wave that moves upstream towards the shock:

$$
R_{D}^{t+\Delta t}=\tilde{a}_{d}^{t+\Delta t}+\frac{\gamma-1}{2} \tilde{\mathbf{u}}_{d}^{t+\Delta t} \cdot \mathbf{n}
$$

In Eq. (14) $\mathbf{n}$ is the shock normal, $\tilde{a}_{d}^{t+\Delta t}$ is the speed of sound and $\tilde{\mathbf{u}}_{d}^{t+\Delta t}$ is the flow velocity on the shock-downstream side of the shock. It is noted that $R_{D}^{t+\Delta t}$ is assumed to be correctly computed by the CFD solver even if the values $\tilde{a}_{d}^{t+\Delta t}$ and $\tilde{\mathbf{u}}_{d}^{t+\Delta t}$ may each be incorrect.

These transfers operators need to be applied twice, once to and once from the shock, and are discussed below. 


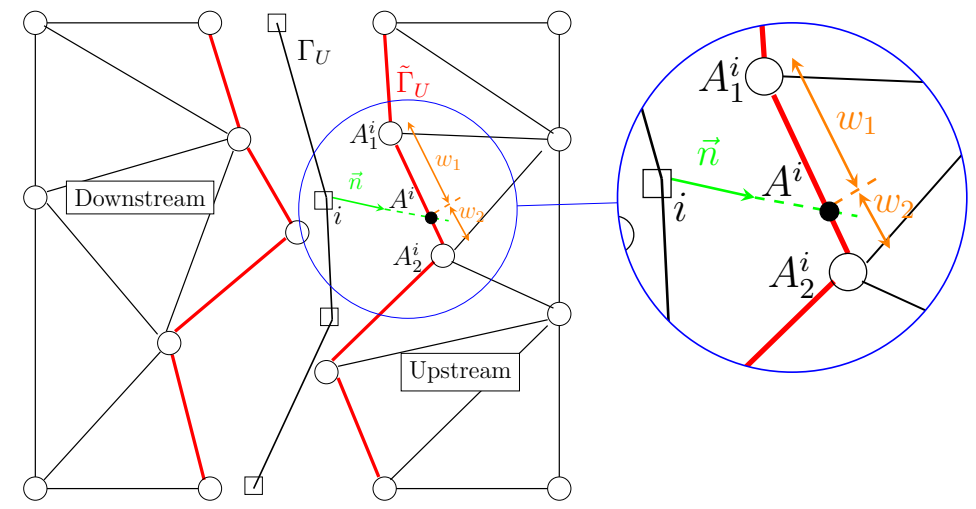

Figure 6: Transfer of variables from the surrogate boundary $\tilde{\Gamma}_{U}$ to the upstream shock state.

\subsection{First transfer: from the surrogate boundaries to the shock}

Since the CFD solver uses Roe's parameter vector $\mathbf{Z}[40]$ as the dependent variable, this is the set of variables used to transfer data between the shock and the surrogate boundaries. The first transfer is required to update the shock-upstream points on $\Gamma_{U}$ and to transfer $R_{D}^{t+\Delta t}$ from $\tilde{\Gamma}_{D}$ to $\Gamma_{D}$.

For both transfers, a Taylor series expansion truncated to the second order is used for the extrapolation:

$$
Z_{i}(\boldsymbol{x})=Z_{i}(\tilde{\boldsymbol{x}})+\nabla Z_{i}(\tilde{\boldsymbol{x}}) \cdot(\boldsymbol{x}-\tilde{\boldsymbol{x}})+o\left(\|\boldsymbol{x}-\tilde{\boldsymbol{x}}\|^{2}\right)
$$

where $Z_{i}$ is any of the four components of $\mathbf{Z}=\sqrt{\rho}(1, H, u, v)^{t}, x$ and $\tilde{x}$ are the coordinates of two different points that belong to $\Gamma$, resp. $\tilde{\Gamma}$, and $\nabla Z_{i}(\tilde{\boldsymbol{x}})$ is the gradient computed on the surrogate boundary, using Eq. (16). Note that, in order to achieve an overall second order of accuracy in the calculation of $Z_{i}(x)$, the approximation of the gradient in Eq. (15) only needs to be consistent, i.e. first-order-accurate.

The first transfer consists in two phases.

1. Upstream: from $\tilde{\Gamma}_{U}$ to $\Gamma_{U}$

The first phase consists in extrapolating from $\tilde{\Gamma}_{U}$ to $\Gamma_{U}$.

In order to be consistent with the physics of the problem, the transfer of variables takes place along the direction of the shock-normal in the shock-point that has to be updated. As shown in Fig. 6, point $A^{i}$ is 
the intersection between the surrogate boundary $\tilde{\Gamma}_{U}$ and the straightline parallel to the shock-normal in shock-point $i$ which passes through shock-point $i$. The value of the dependent variables (and their gradients) in point $A^{i}$ is computed using the solution in grid-points $A_{1}^{i}$ and $A_{2}^{i}$ by means of the following formula:

$$
\phi\left(A^{i}\right)=\phi\left(A_{1}^{i}\right) w_{2}+\phi\left(A_{2}^{i}\right) w_{1}
$$

where $\phi$ is either $Z_{i}$ or $\nabla Z_{i}$, and $w_{1}$ and $w_{2}$ are the weights, equal to the normalized distances between $A^{i}$ and grid-points $A_{1}^{i}$ and $A_{2}^{i}$. When Eq. (16) is used to compute the gradient, the evaluation of the gradient in the grid-points of the surrogate boundaries is performed here using an area-weighted formula, which is reported in Appendix 1.

Once the value of $\mathbf{Z}$ in the intersection point $A^{i}$ has been computed using Eq. (16) the value of $\mathbf{Z}$ in shock-point $i$ is computed by means of Eq. (15), having set $x$ equal to the coordinates of shock-point $i$ and $\tilde{x}$ to those of $A^{i}$.

\section{Downstream: from $\tilde{\Gamma}_{D}$ to $\Gamma_{D}$ :}

The Riemann variable defined by Eq. (14), which is the only quantity that has been correctly computed on $\tilde{\Gamma}_{D}$ by the unstructured shockcapturing solver, has to be transferred from $\tilde{\Gamma}_{D}$ to $\Gamma_{D}$ using Eq. (15). The procedure is identical to that used for the upstream boundaries: starting from the shock-point to be updated and moving forward along its normal vector as far as an edge of $\tilde{\Gamma}_{D}$ is intersected in $B^{i}$ (see Fig. 7).

\subsection{Shock calculation}

As already mentioned and also schematically shown in Fig. 5, each shockpoint consists in two superimposed grid-points, which represent the shockupstream and the shock-downstream states. The velocity component in the shock-normal direction, $w$, is also stored within each shock-point. For the reasons explained in step 5.6, the shock-upstream state and the Riemann variable $R_{D}$, Eq. (14), on the shock-downstream side of the shock have been correctly updated at time level $t+\Delta t$. The shock-downstream state $\left(\rho_{d}, p_{d}\right.$ and $\mathbf{u}_{d}$ ) and the shock-speed at time $t+\Delta t$, which are yet unknown at this stage, are found by solving a system of five algebraic non-linear equations. 


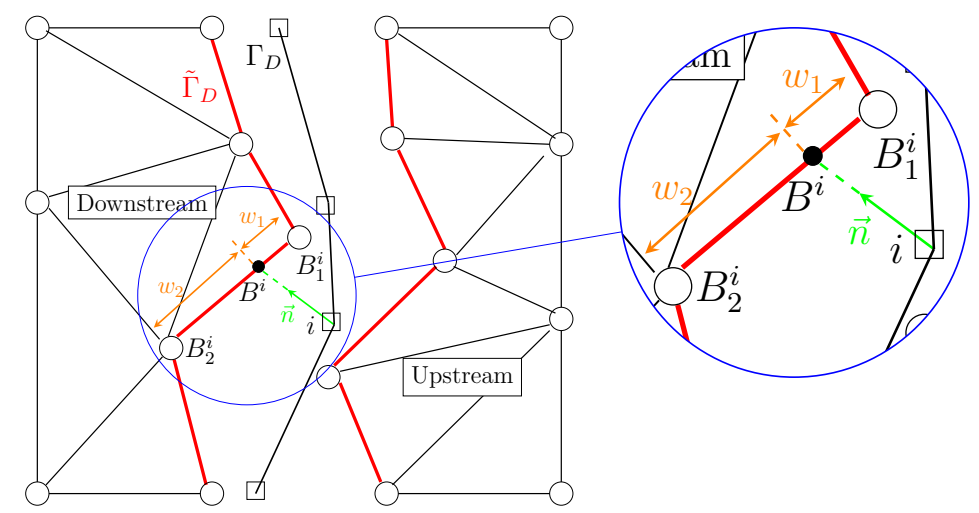

Figure 7: Transfer of the Riemann's variable from the surrogate boundary $\tilde{\Gamma}_{D}$ to the shock point.

The first four equations are the Rankine-Hugoniot jump relations and the fifth is Eq. (14):

$$
\begin{aligned}
\rho_{d}^{t+\Delta t}\left(\mathbf{u}_{d}^{t+\Delta t} \cdot \mathbf{n}-w\right) & =\rho_{u}^{t+\Delta t}\left(\mathbf{u}_{u}^{t+\Delta t} \cdot \mathbf{n}-w\right) \\
\rho_{d}^{t+\Delta t}\left(\mathbf{u}_{d}^{t+\Delta t} \cdot \mathbf{n}-w\right)^{2}+p_{d}^{t+\Delta t} & =\rho_{u}^{t+\Delta t}\left(\mathbf{u}_{u}^{t+\Delta t} \cdot \mathbf{n}-w\right)^{2}+p_{u}^{t+\Delta t} \\
\frac{\gamma}{\gamma-1} \frac{p_{d}^{t+\Delta t}}{\rho_{d}^{t+\Delta t}}+\frac{1}{2}\left(\mathbf{u}_{d}^{t+\Delta t} \cdot \mathbf{n}-w\right)^{2} & =\frac{\gamma}{\gamma-1} \frac{p_{u}^{t+\Delta t}}{\rho_{u}^{t+\Delta t}}+\frac{1}{2}\left(\mathbf{u}_{u}^{t+\Delta t} \cdot \mathbf{n}-w\right)^{2} \\
\mathbf{u}_{d}^{t+\Delta t} \cdot \tau & =\mathbf{u}_{u}^{t+\Delta t} \cdot \tau \\
R_{D}^{t+\Delta t} & =\tilde{a}_{d}^{t+\Delta t}+\frac{\gamma-1}{2} \tilde{\mathbf{u}}_{d}^{t+\Delta t} \cdot \mathbf{n}
\end{aligned}
$$

Hence, for system (17), the vector of known variables $\left(\rho_{u}, p_{u}, \mathbf{u}_{u}, R_{D}\right)$ is then used to find the updated values of the unknowns ones $\left(\rho_{d}, p_{d}, \mathbf{u}_{d}\right.$, $w)$. The system (17) is solved within each shock-point using the NewtonRaphson root-finding algorithm, thus providing the correct downstream state and shock-speed at time level $t+\Delta t$.

\subsection{Second transfer: from the shock to the surrogate boundaries}

Once the shock-downstream states along the shock-boundary $\Gamma_{D}$ have been updated as described in step 5.7, the grid-points on the downstream surrogate boundary $\tilde{\Gamma}_{D}$ need also to be updated.

Downstream: from $\Gamma_{D}$ to $\tilde{\Gamma}_{D}$

The first step needed to update grid-point $i$ on $\tilde{\Gamma}_{D}$ consists in finding the projection $P^{i}$ of grid-point $i$ on the shock-poly-line. This is accomplished by 


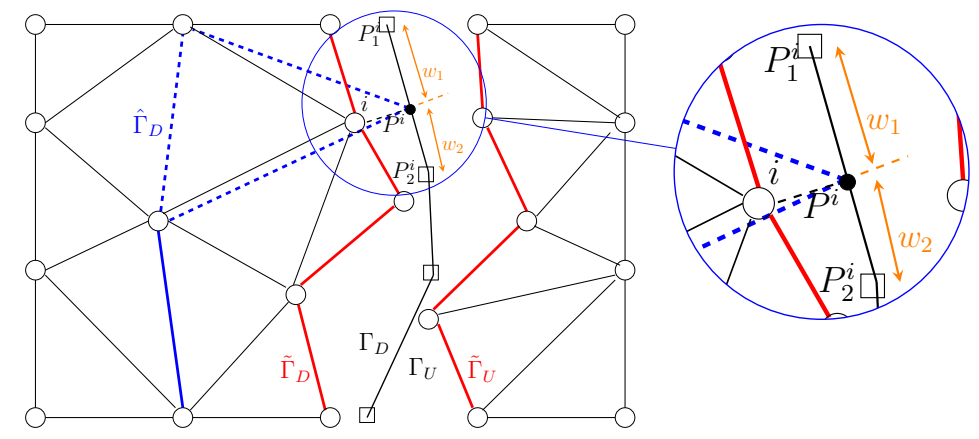

Figure 8: Search of the auxiliary point $P^{i}$ on the shock poly-line used to interpolate the dependent variables in the grid-points of the surrogate boundary $\tilde{\Gamma}_{D}$.

first locating the closest shock-edge to grid-point $i$ and then projecting along the direction which is the weighted average ${ }^{2}$ of the two vectors normal to the shock in $P_{1}^{i}$ and $P_{2}^{i}$. Then, the dependent variables in $P^{i}$ are computed using Eq. (16), the weights $w_{1}$ and $w_{2}$ being the normalized distances of $P^{i}$ from shock-points $P_{1}^{i}$ and $P_{2}^{i}$.

The second step consists in using point $P^{i}$ and two grid-points that belong to the second surrogate boundary $\hat{\Gamma}_{D}$ to build a triangle (shown using a dashed blue line in Fig. 8), which contains grid-point $i$.

Finally, the dependent variables in grid-point $i$ are linearly interpolated within that triangle.

The reason for using a second surrogate boundary on the downstream side of the shock lies in fact that, whenever the shock-downstream flow is subsonic, the acoustic waves spread in all directions. Under this circumstance, only grid-points (such as those on $\hat{\Gamma}_{D}$ ) that are surrounded on all sides by cells have been correctly updated by the CFD solver.

\subsection{Shock displacement}

The new position of the shock-front at time level $t+\Delta t$ is computed by displacing all shock-points using the following first-order-accurate (in time) formula:

$$
\mathbf{x}\left(P^{t+\Delta t}\right)=\mathbf{x}\left(P^{t}\right)+w^{t+\Delta t} \mathbf{n} \Delta t
$$

\footnotetext{
${ }^{2}$ the weights depend upon the normalized distance between the two shock-points $P_{1}^{i}$ and $P_{2}^{i}$ and grid-point $i$.
} 


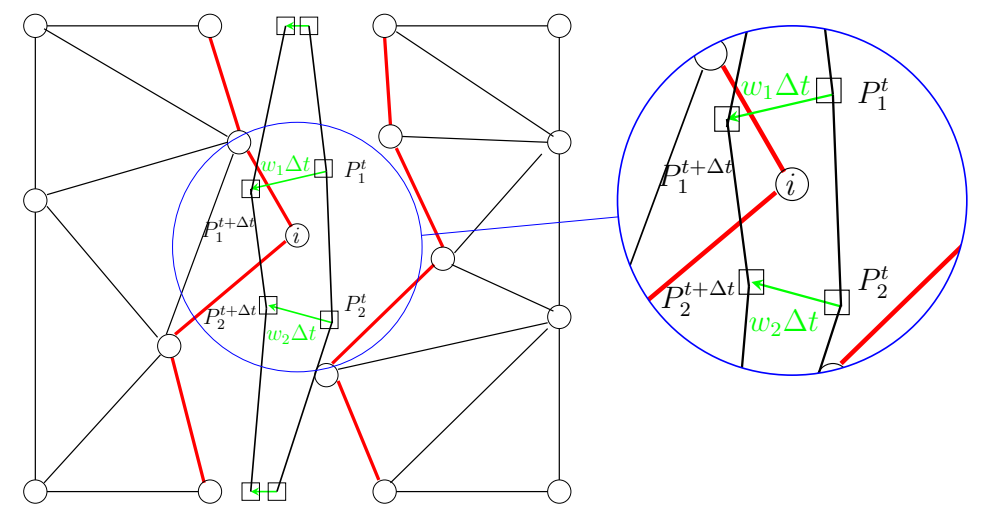

Figure 9: The shock-front overtakes a grid-point of the background mesh during its motion.

where $\mathbf{x}(P)$ denotes the geometrical location of the shock-points. The use of a first-order-accurate temporal integration formula in (18) is immaterial as long as steady flows are of interest. In this case, a second-order accurate representation of the shock-shape is guaranteed by the use of second-orderaccurate formulae to compute the shock-normal, as described in step 5.3. For unsteady flows, second-order-accurate time integration formulae should be used, as done for example in [11, 12]. As can be seen from Fig. 9, the shockfront can freely float over the background triangulation and, while doing so, it may cross the downstream surrogate boundary. This is the situation sketched in Fig. 9, where the shock-fronts at time level $t$ and $t+\Delta t$ have both been drawn. In the sketch of Fig. 9, grid-point $i$ has been overtaken by the moving shock-front. Whenever this happens, the flow state within grid-point $i$ should be changed accordingly. This is the task performed in the next step.

\subsection{Re-interpolation of nodes crossed by the shock}

This step of the algorithm consists in the interpolation of those grid-points of the background mesh that have been overtaken by the shock-front, thus passing from one region to the other. In order to understand whether gridpoint $i$ has been overtaken or not by the shock, the position of the closest shock-edge, before and after the displacement, i.e. at time $t$ and $t+\Delta t$, is used to build a quadrilateral, as shown in Fig. 9. If grid-point $i$ falls inside the quadrilateral of vertices $P_{1}^{t}, P_{2}^{t}, P_{1}^{t+\Delta t}$ and $P_{2}^{t+\Delta t}$, grid-point $i$ has been overtaken and its state has to be updated. The state of grid-point $i$ is updated 


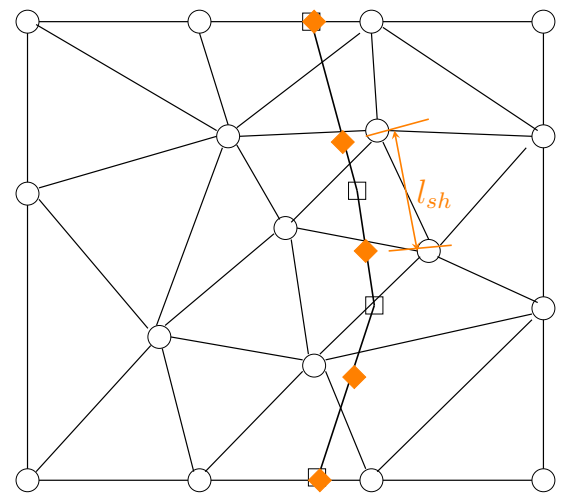

Figure 10: Shock-point location along the shock front: before (squares) and after (diamond) the re-distribution step.

using an interpolation procedure similar to that illustrated in step 5.8 .

\subsection{Shock-point re-distribution}

During the shock displacement step, the shock-edges may stretch or shorten, depending on the relative motion of the various shock-point that make up the shock-front. This might lead to a shock-poly-line made of shock-edges whose length is considerably different from the local size of the background mesh. To avoid such a risk, a shock-point re-distribution can be performed as the last step of the algorithm. Doing so, it is possible to ensure that the shock-edge lengths are approximately equal to the edges of the underlying background mesh. A naive shock-point re-distribution procedure is done by imposing that all shock-edges have the same fixed length $l_{s h}$, preset by the user. Whenever the shock-points are re-located along the shock-front, both the shock-upstream and shock-downstream state within each shockpoint have to be re-computed, a task which is easily accomplished using linear interpolation along the shock-front. Figure 10 shows the location of the shock-points along the shock-front both before and after the re-distribution. At this stage, the numerical solution has been correctly updated at time level $t+\Delta t$.

\section{Numerical results}

All physical quantities displayed in this section have been made dimensionless using the following set of reference variables: $L, \rho_{\infty}, \mathbf{u}_{\infty}$, where $L$ is 
a length scale, $\rho_{\infty}$ and $\mathbf{u}_{\infty}$ the free-stream density and flow speed. Using the aforementioned set of reference variables, the reference pressure is twice the free-stream dynamic pressure: $\rho_{\infty}\left(\mathbf{u}_{\infty} \cdot \mathbf{u}_{\infty}\right)$.

\subsection{Quasi-one-dimensional nozzle flow}

The quasi-one-dimensional (Q1D) steady flow through a variable area duct (converging-diverging nozzle) turns out to be particularly well suited as a validation case because the flow is non-uniform both upstream and downstream of the shock and an analytical solution is available, which allows to compute the discretization error, $\epsilon$, i.e. the difference between the exact and computed solutions. Moreover, a similar study reported in [41], showed that the discretization error within the entire shock-downstream region exhibits first-order convergence as the grid is refined even if high-order-accurate schemes are used. This is a known deficiency of shock-capturing schemes which we will show does not affect the eST method.

The nozzle geometry has been taken from [41]:

$$
A / A_{*}=1+\left(A_{e} / A_{*}-1\right)(x / L)^{2} \quad \text { where } \quad-1 / 2 \leq x / L \leq 1
$$

and the exit-to-sonic area ratio is equal to $A_{e} / A_{*}=2$. Having set the ratio between the exit-static to inlet-total pressures equal to $p_{\text {out }} / p_{\text {in }}^{0}=0.7362$, a steady normal shock occurs in the diverging part of the nozzle at about $x_{s h} / L=0.75$.

In order to simplify the treatment of the boundary conditions, the left boundary of the computational domain has been set at $x_{\text {left }} / L=0.05$, just downstream of the troath, where a supersonic inflow boundary condition applies.

The main advantage of the present test-case is the fact that it has an analytical solution. In particular, the Mach number distribution follows from the so-called area-rule:

$$
\frac{1}{M}\left[\frac{2}{\gamma+1}\left(1+\frac{\gamma-1}{2} M^{2}\right)\right]^{\frac{\gamma+1}{2(\gamma-1)}}=\frac{A}{A^{*}}
$$

A comparison has been made between the SC and eST simulations using a sequence of uniformly spaced grids, with grid densities ranging between 800 and 6400 cells, see Tab. 1.

Knowledge of the exact solution allows to compute (rather than estimate) the discretization error and, therefore, to perform reliable convergence tests. 


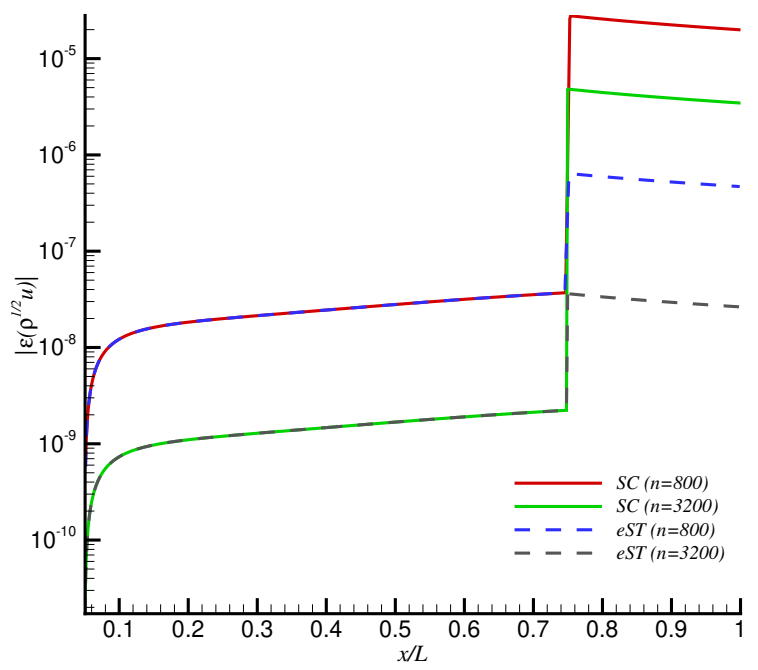

Figure 11: Q1D nozzle flow: pointwise errors analysis for SC and eST.

Figure 11 shows the pointwise distribution of the discretization error for the third component of Roe's parameter vector. Note that the y-axis of Fig. 11 is in logarithmic scale. As expected, the SC and eST simulations feature the same discretization error in the entire supersonic, shock-upstream region. Downstream of the shock, however, SC incurs in a discretization error which is about two orders of magnitude larger than that of the eST. The accuracy degradation incurred by SC within the entire downstream region is further confirmed in Fig. 12, which shows, in a log-log scale, the $L_{1}$ norm of the

Table 1: Q1D nozzle flow: characteristics of the background meshes used to perform the grid-convergence tests.

\begin{tabular}{crc}
\hline \hline Grid level & Cells & $h$ \\
\hline 0 & 800 & $1.87510^{-3}$ \\
1 & 1600 & $9.37510^{-4}$ \\
2 & 3200 & $4.68810^{-4}$ \\
3 & 4800 & $3.12510^{-4}$ \\
4 & 6400 & $2.34410^{-5}$ \\
\hline \hline
\end{tabular}




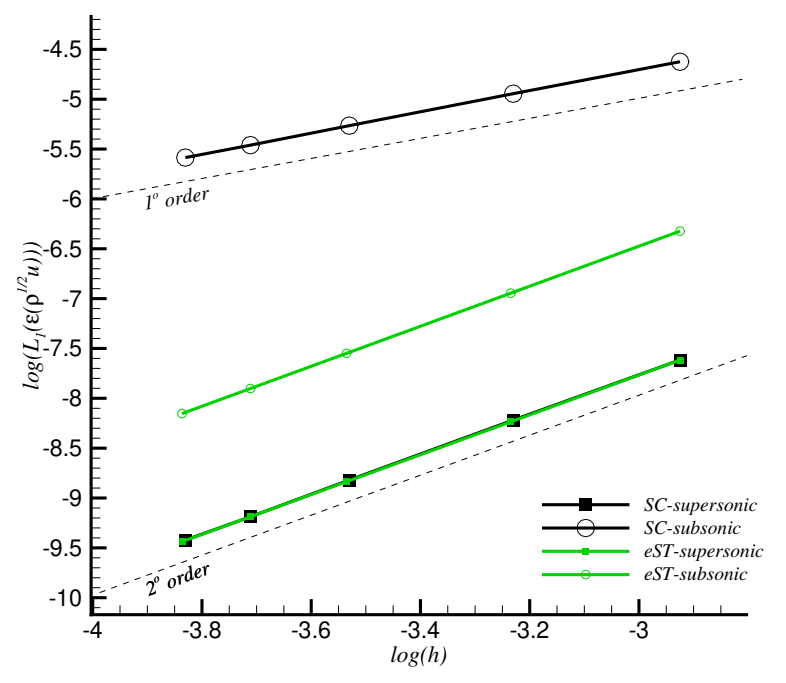

Figure 12: Q1D nozzle flow: global measures of the discretization error for SC and eST.

discretization error plotted against the mesh spacing. In contrast to the pointwise measure displayed in Fig. 11, Fig. 12 shows a global measure, which has been separately computed within the shock-upstream $\left(x_{L} \leq x<x_{s h}\right)$ and shock-downstream $\left(x_{s h}<x \leq L\right)$ sub-domains. The difference between the error-reduction trends exhibited by SC and eST is striking: the two different shock-modeling practices behave identically within the shock-upstream subdomain, where both exhibit second-order convergence as the mesh is refined; within the shock-downstream sub-domain, however, eST retains second-order convergence, whereas SC drops to first-order.

\subsection{Planar source flow}

This test case consists in a compressible, planar source flow that has already been studied in $[42,43]$ as a validation case, due to the availability of an analytical solution. Indeed, assuming that the analytical velocity field has a purely radial velocity component, it may be easily verified that the governing PDEs, written in a polar coordinate system, become identical to those governing a compressible quasi-one-dimensional variable-area flow (20), provided that the nozzle area varies linearly with the radial distance, $r$, from 


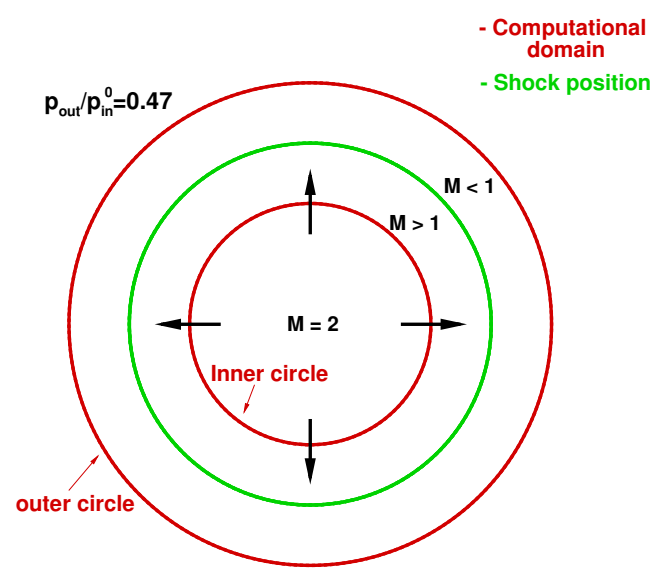

(a) Sketch of the computational domain.

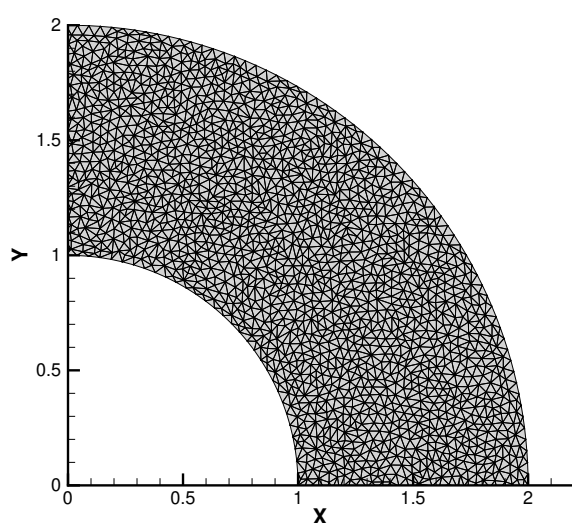

(b) Detail of the unstructured mesh employed for the simulations.

Figure 13: Planar, transonic source flow.

the pole of the reference frame. The computational domain consists in the annulus sketched in Fig. 13a: the ratio between the radii of the outer and inner circles $\left(L=r_{i n}\right)$ has been set equal to $r_{\text {out }} / r_{i n}=2$. A transonic (shocked) flow has been studied by imposing a supersonic inlet flow at $M=$ 2 on the inner circle and a ratio between the outlet static and inlet total pressures $p_{\text {out }} / p_{\text {in }}^{0}=0.47 \mathrm{such}$ that the shock forms at $r_{s h} / r_{i n}=1.5$. The Delaunay mesh shown in Fig. 13b, which contains 6916 grid-points and 13456 triangles, has been generated using triangle $[44,45]$ in such a way that no general alignment is present between triangle edges and shock, thus making the discrete problem truly two-dimensional. Figure 14 shows a comparison between the SC and eST solutions, both in terms of entropy, $S=p \rho^{-\gamma}$, and $\sqrt{\rho} u$ iso-lines. Both flow variables clearly reveal that the SC solution is plagued by severe spurious errors due to the misalignment between the edges of the mesh and the captured shock. These errors propagate in the shock-downstream region, as it is evident from the entropy field of the SC calculation, compromising the quality of the solution. Note that across the numerical shock layer obtained with the SC approach, the direction of the velocity vector is undefined and largely dependent on the mesh topology. This explains the perturbations observed in the shock-downstream region.

Thanks to the availability of the analytical solution, a point-wise error 


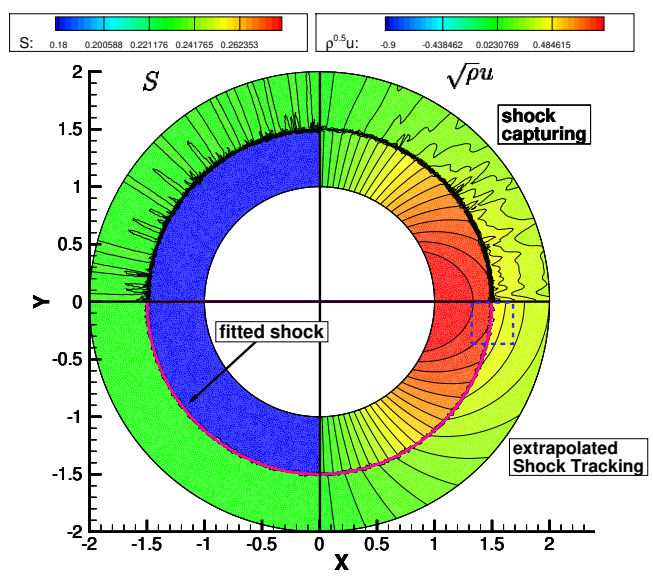

(a)

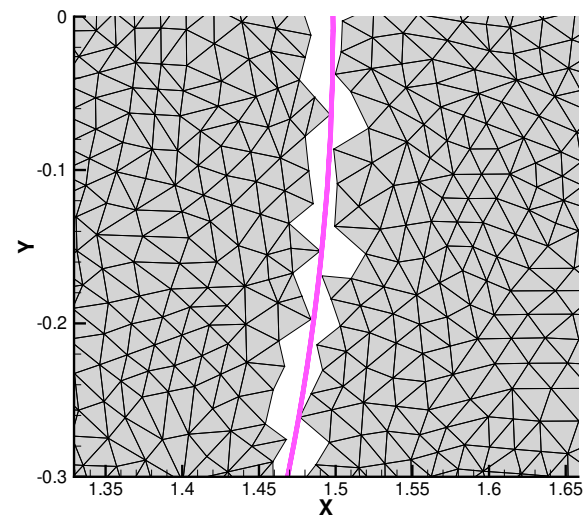

(b)

Figure 14: Planar source flow. (a): entropy (left half of the frame) and third component $(\sqrt{\rho} u)$ of $\mathbf{Z}$ (right half of the frame). SC result on the top, eST result on the bottom. (b): close-up of the blue square drawn in frame (a).

analysis has been carried out by computing the discretization error. Figure 15 shows the behavior of the local discretization error in all points of the mesh, plotted against the radial distance from the center of the circle. The vertical line drawn in Fig. 15 points to the position $(r=1.5)$ where the shock-wave takes place. It can be seen that upstream of the shock the error of the SC and eST solutions is equal. Downstream of the shock $(r>1.5)$, however, the eST solution exhibits an error which is one or two orders of magnitude lower than that obtained using SC.

An order-of-convergence analysis, similar to that of Sect. 6.1, has also been performed by repeating the same calculation on three nested triangulations whose features are summarized in Tab. 2 , where $h$ is the mesh spacing along the inner and outer circular boundaries. The coarsest mesh is the one shown in Fig. 13b and the two finer meshes have been obtained by recursively subdividing each triangle of the parent mesh into four nested triangles.

A global measure of the discretization error has been computed using the $L_{1}$-norm of $\epsilon(\sqrt{\rho} v)$ and $\epsilon(\sqrt{\rho} H)$, separately within the shock-upstream and shock-downstream sub-domains; the results are displayed in Fig. 16 and also include those published in [42], which have been obtained using the unstructured shock-fitting technique developed by some of the authors in [8]. It is noted that within the supersonic, shock-upstream region, the three numeri- 


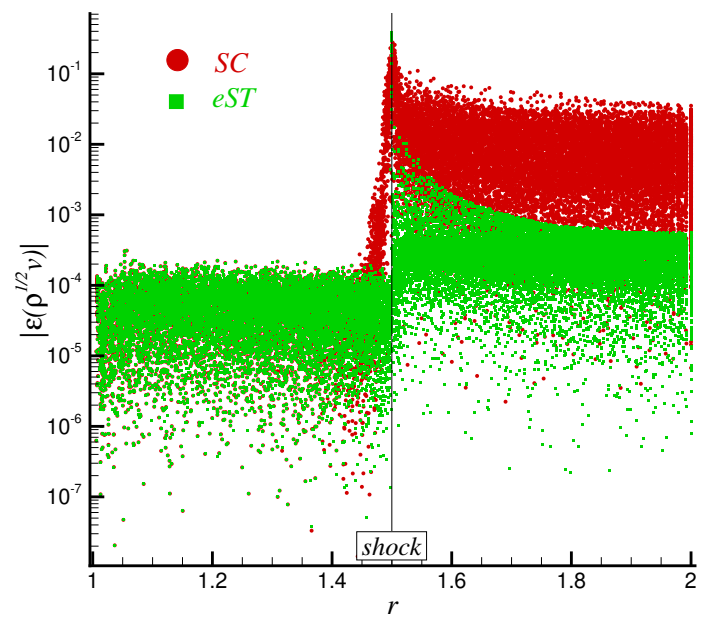

Figure 15: Planar, transonic source flow: pointwise error analysis (SC vs. eST).

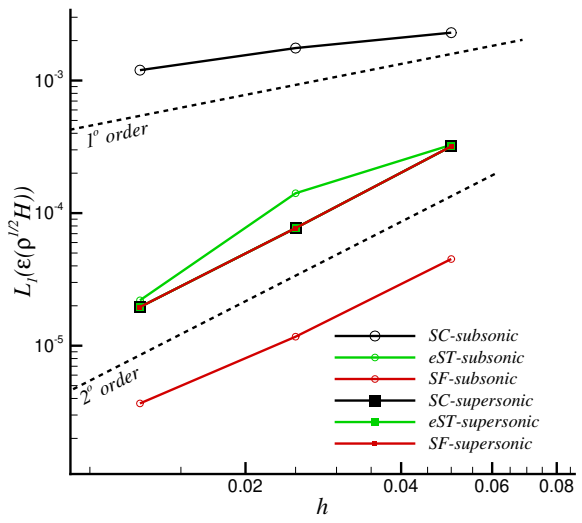

(a) $\sqrt{\rho} H$

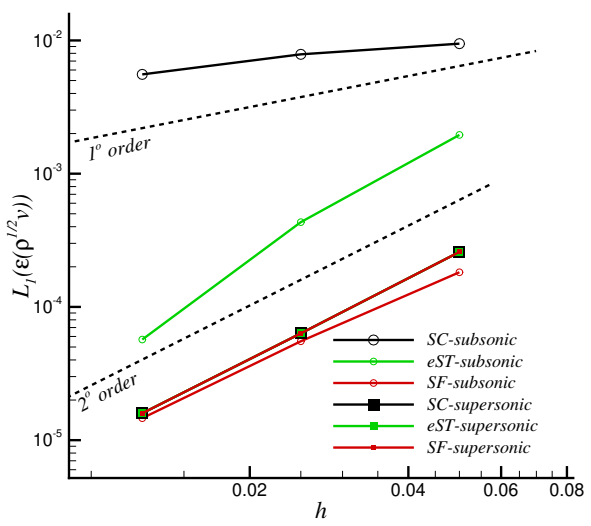

(b) $\sqrt{\rho} v$

Figure 16: Planar, transonic source flow: order-of-convergence comparison among SC, eST and SF w.r.t the second and fourth components of the parameter vector $\mathbf{Z}$. 
Table 2: Planar source flow: characteristics of the background meshes used to perform the grid-convergence tests.

\begin{tabular}{crcr}
\hline \hline Grid level & Grid-points & Triangles & $h$ \\
\hline 0 & 6,916 & 13,456 & 0.05 \\
1 & 27,288 & 53,824 & 0.025 \\
2 & 108,400 & 215,296 & 0.0125 \\
\hline \hline
\end{tabular}

cal solutions feature the same discretization error and converge to the exact solution at design (second) order as the mesh is refined. Downstream of the shock, however, only the two shock-fitting techniques (SF and eST) exhibit second-order convergence, whereas SC has fallen below first-order. Finally, the comparison between the SF technique of [8] and the eST technique described here reveals that the latter incurs in a slightly larger discretization error than the former within the shock-downstream region. This observation points to the fact that there is room for improving the data transfer algorithms described in Sect. 5.6 and 5.8, which will be the subject of future work.

\subsection{Cost vs. accuracy analysis}

A comparative assessment of the computational cost of the shock-fitting and shock-capturing approaches can be made by either: i) using the same meshes, or ii) estimating the (different) mesh spacing required by the two techniques to achieve the same discretization-error level in the shock-downstream region.

If the first standpoint is adopted, i.e. the same grids are used, it is clear that shock-fitting techniques, thus including both SF and eST, incur an higher computational cost per time-step than SC. This is because, in addition to solving the governing PDEs on the same mesh, using the same CFD solver also used in the SC simulation, shock-fitting techniques also have to keep track of the shock motion by solving the Rankine-Hugoniot relations at all shock-points. However, since the shock-mesh has a lower dimensionality $(d-1$ in the $d$-dimensional space) than the mesh that fills the computational domain, the overall increase in computational cost incurred by either the SF or eST techniques amounts to a relatively small fraction of the cost per iteration of the SC technique. The interested reader can find a detailed analysis on the computational costs incurred by the SF technique in [46]. 
If, on the contrary, the second standpoint is adopted, shock-fitting methods are seen to outperform shock-capturing when it comes to achieve the same discretization error level in the shock-downstream region. The idea here is to use the results of the grid convergence tests of Sect. 6.1 and 6.2 to estimate the mesh sizes required by the SC solver to provide error levels comparable to those of the eST approach. The analysis described below is based on the discretization error of the third component $\epsilon(\sqrt{\rho} u)$ of $\mathbf{Z}$ for the Q1D nozzle flow, whereas the fourth component $\epsilon(\sqrt{\rho} v)$ has been considered on two-dimensional grids.

The computations of the Q1D nozzle-flow of Sect. 6.1 show that using the SC solver, which has a shock-downstream convergence rate of about 1.1, see Fig. 12, a mesh size $h \simeq 3.7510^{-5}$ would be required to obtain the same error provided by the eST approach on the coarsest, level 0 mesh, see Tab. 1. This amounts to say that in $1 \mathrm{D}$ SC requires a mesh that is about $32\left(=h_{0} / h\right)$ times finer than the level 0 mesh to obtain the coarse-grid eST result. Note that $h$ is even smaller than the mesh spacing $h_{4}=2.34410^{-4}$ (see Tab. 1) of the finest mesh used in the grid-convergence study and that such a fine mesh would be required just to compensate the error generated by capturing the shock. Moreover, in order to obtain the same discretization error of the eST approach on the finest (level 4) mesh, the SC solver would need a mesh size $h^{\prime} \simeq 2.7310^{-7}$, which is three orders of magnitude smaller than $h_{4}$.

The two-dimensional source flow computations of Sect. 6.2 show that in order to obtain the same discretization error of eST on the coarsest, level 0 mesh, SC would require a mesh spacing $h \simeq h_{0} / 64$, whereas to attain an error level comparable to that obtained by eST on the finest, level 2 mesh, SC would require a mesh spacing $h^{\prime} \simeq 1.5310^{-6}$, which is roughly four orders of magnitude smaller than $h_{2}$, see Tab. 2 .

These results give indications that using uniform refinement in $2 \mathrm{D}$, the same error level of the coarse-grid, eST result would be attained with SC using a mesh having a number of triangular elements that is $\left(h_{0} / h\right)^{2} \simeq 4096$ times larger than the number of triangles of the level 0 mesh, see Tab. 2. This amounts to a number of triangular cells of the order of $10^{7}$. Following the same line of reasoning, obtaining the fine-grid, eST result using SC would instead require a $2 \mathrm{D}$ mesh with a number of elements that is $\left(h_{2} / h^{\prime}\right)^{2} \simeq 10^{8}$ times larger than the level 2 grid, which is clearly impractical. A possible solution would be to replace eST by some aggressive error estimation and anisotropic metric-based adaptation techniques, as e.g. those proposed in [47]. However, one should evaluate the capabilities of these techniques 


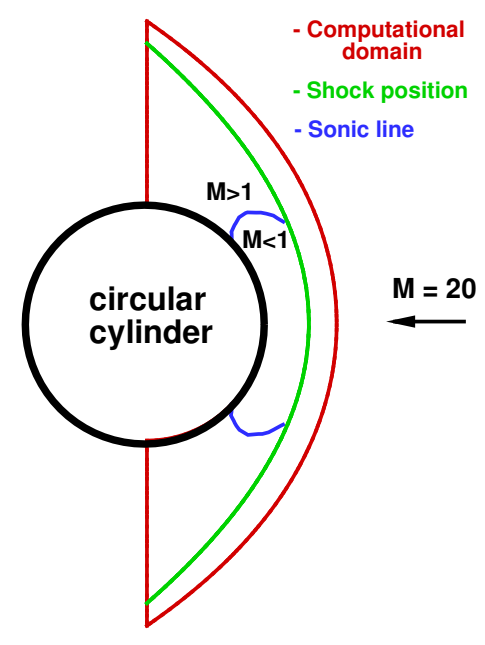

Figure 17: Hypersonic flow past a circular cylinder: sketch of the computational domain.

to provide meshes with the anisotropy ratios required to drop the mesh size down several order of magnitudes in the shocks, the capabilities of the flow solver involved to handle such meshes, and, finally, the overhead of the mesh refinement itself compared to that of the eST method. This is perhaps a possible avenue for future work.

\subsection{Blunt body problem}

The hypersonic $\left(M_{\infty}=20\right)$ flow past the fore-body of a circular cylinder, see Fig. 17, is a comprehensive test-bed for the eST algorithm, because the entire shock-polar is swept whilst moving along the bow shock which stands ahead of the blunt body. The existence of the subsonic pocket that surrounds the stagnation point and the transition to supersonic flow through the sonic line may be challenging for the proposed method and, in particular, for the algorithms used to transfer data back and forth between the shock and surrogate boundaries.

The mesh used as the background triangulation in the eST simulation has also been used to run the SC simulation. The computations have been run on a Delaunay mesh containing 808 points and 1458 triangles, generated using delaundo $[48,49]$. A close up view of the mesh is reported on Fig. 18: it can be seen that the only difference between the eST and SC computation 


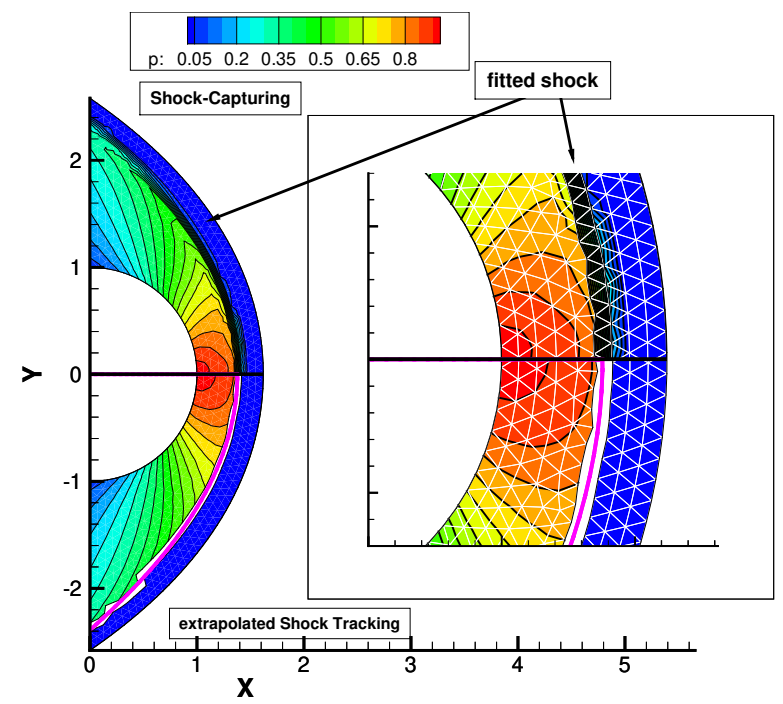

Figure 18: Hypersonic flow past a circular cylinder: comparison between the pressure iso-contours computed by means of SC (top) and eST (bottom). Computed shock curve in pink.

consists in the removal of the triangles crossed by the fitted-shock in the eST case.

Pressure iso-contour lines are shown in Fig. 18: the SC calculation is shown in the upper half of both frames and the eST one in the lower half. The steady location of the fitted bow shock (shown using a solid bold line) has also been superimposed on both the SC and eST results. The comparison clearly reveals that the differences between the solutions obtained using the two different shock-modeling practices are remarkable within the entire shocklayer.

Figure 19 shows the pressure $p$ profile probed along a line that makes a $45^{\circ}$ angle w.r.t. the centerline. The SC and eST results have been compared with the reference solution computed in [50]. The comparison shows that the finite shock-width of the SC solution is replaced by a discontinuity in the eST result (which also includes the shock-upstream and shock-downstream values) and that the shock stand-off distance computed by eST agrees very well with the reference solution.

Finally, Fig. 20, which compares the density iso-contour lines computed by the SF technique of Ref. [8] and the eST technique described here, turns 


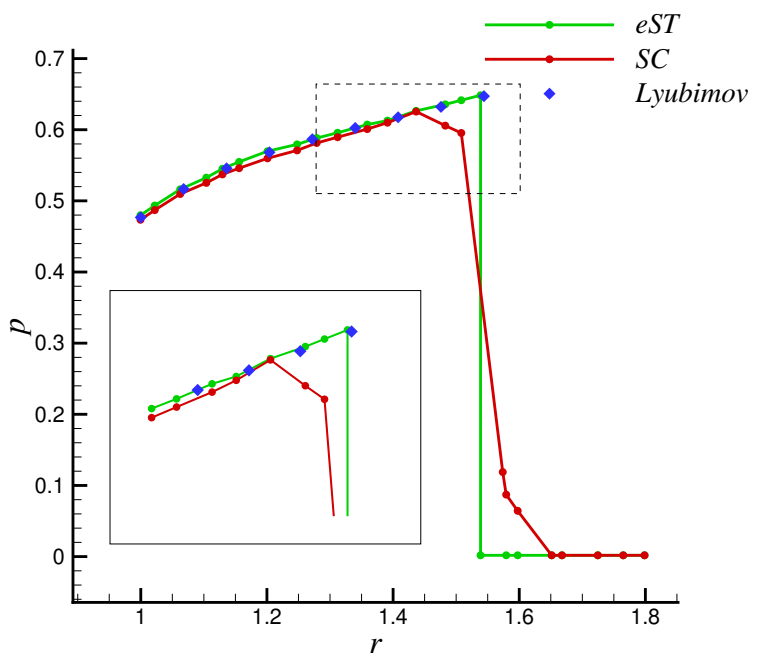

Figure 19: Hypersonic flow past a circular cylinder: SC, eST and reference [50] pressure distribution within the shock-layer.

out to be very useful to pinpoint the methodological differences between the two different shock-fitting approaches. Observe, in particular, that in the eST simulation the solution has not been computed within the blank region surrounding the fitted shock (for clarity, the fitted-shock has not been drawn in Fig. 20b). Even so, the eST solution within the shock-layer is as smooth as it is the one computed by SF.

\subsection{Hybrid computations of interactions}

In its current implementation, the eST method cannot explicitly track shock interactions. However, in this section we will show that it can be applied without any problem to this type of flows by means of a hybrid fitcapture approach. We will in particular consider two applications: a steady Mach reflection in a channel with a ramp, and a type IV shock-shock interaction arising in a supersonic flow around a circular cylinder.

Steady Mach Reflection. This case is quite useful as it involves a relatively simple flow pattern, but allows to clearly visualize the advantage brought by the eST approach w.r.t. SC. 


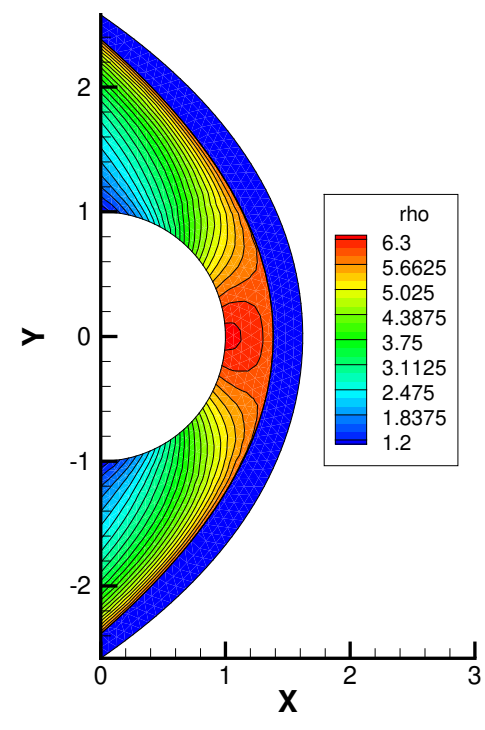

(a) $\mathrm{SF}$

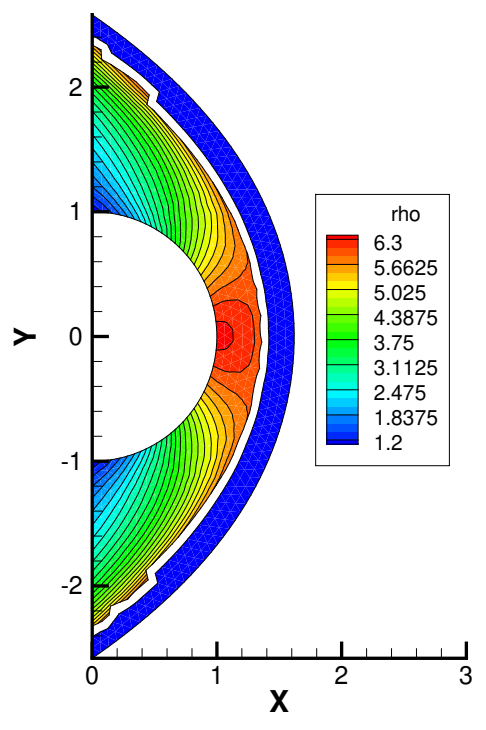

(b) eST

Figure 20: Hypersonic flow past a circular cylinder: comparison between SF and eST in terms of density iso-contour lines.

The set up of the test case is the same as in [8] and sketched on Fig. 21: it involves a $M_{\infty}=2$ flow in a channel with a wall deflection of 14 degrees. The oblique shock forming due to this deflection reflects onto the channel walls. In these conditions the reflection is not a regular one, but a Mach reflection is observed with its typical lambda-shock topology. A sketch of the resulting interaction is reported in Fig. 21. Note that, as a result of this interaction, a contact discontinuity emanates from the triple point.

The background mesh used for this simulation contains 14833 grid-points and 29214 triangles. We compare on this mesh the SC solution with the hybrid result in which eST is only applied to two of the branches of the lambda shock: the Mach stem and the reflected shock. Both the incident shock and the contact discontinuity are captured. Figure 22 displays the Mach iso-contours in the entire computational domain (the left frame), an enlargement of the region surrounding the triple point (the middle frame) and the Mach number distribution along a vertical line at $x=1.5$ (the right frame) in the region downstream of the triple point. It can be seen in Fig. 22, 


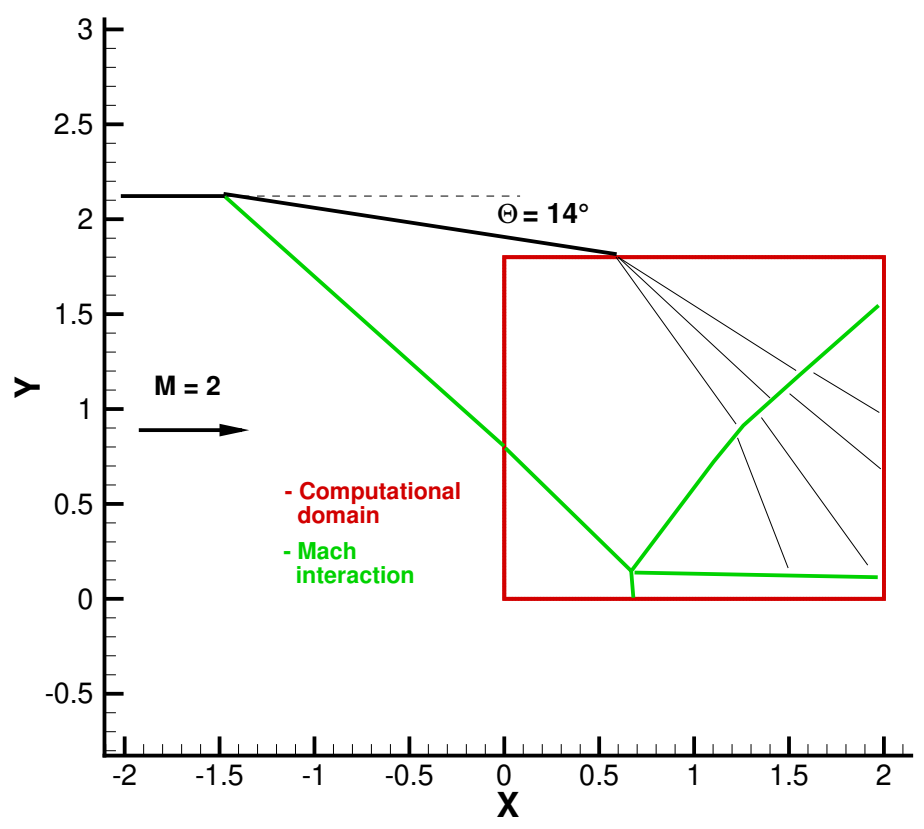

Figure 21: Steady Mach reflection: sketch of the computational domain.

that the capture of the Mach stem gives rise to an unphysical behavior of the Mach number contour lines in the region downstream of the Mach stem. This unphysical behavior disappears in the hybrid solution that exhibits a smoother Mach number distribution. It must be noticed that the gradient reconstruction technique, described in Appendix A, does not provide accurate gradient reconstruction for discontinuous solutions. Nonetheless, this is unlikely to significantly affect the overall quality of the eST computations because only very few grid-points are involved in the part of the domain where the interaction occurs.

Finally, Fig. 23, stands out, again, that the solution obtained with the eST algorithm is notable and comparable with the one described in [8].

Type IV shock-shock interaction. This last benchmark introduced in [51] involves a more complex pattern of interacting discontinuities. An horizontal flow, characterized by a Mach number of $M=5.05$, is deflected by an oblique shock (whose angle w.r.t. the horizontal direction is $\Theta=13$ degrees ) in front 

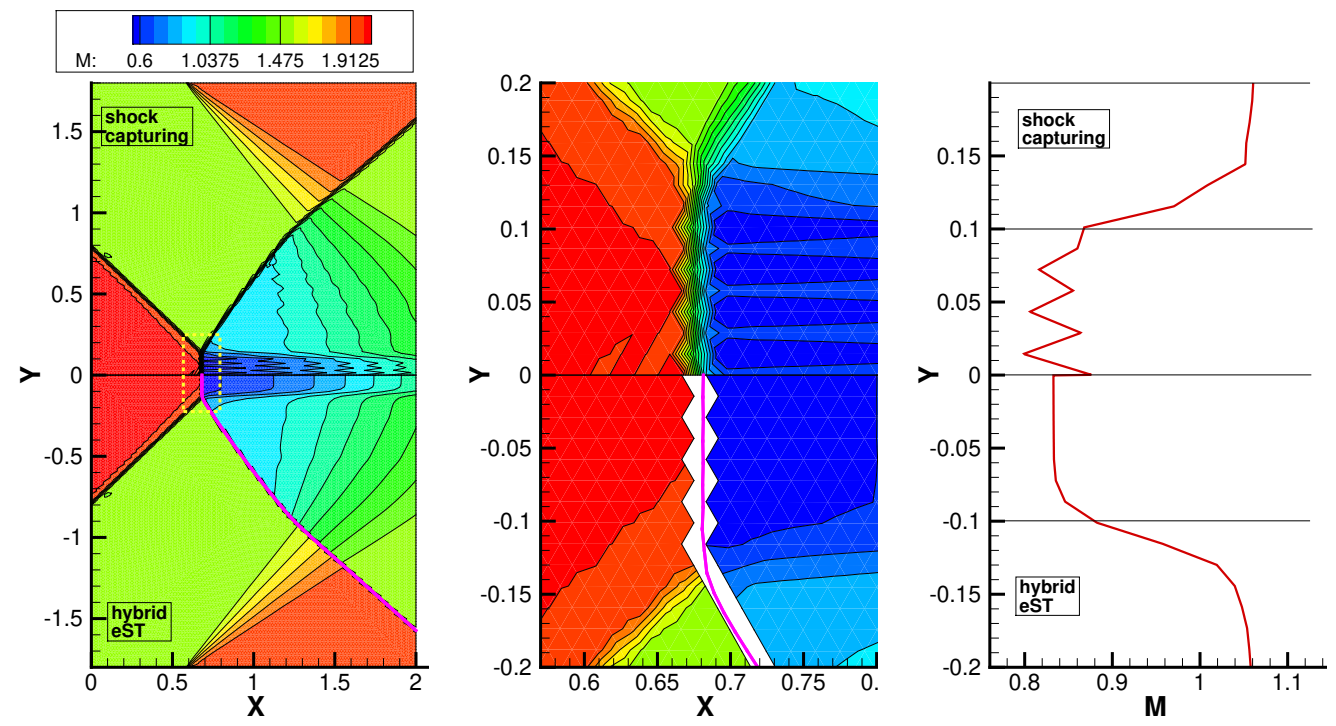

Figure 22: Steady Mach reflection: Mach number iso-contours comparison, enlargement around the triple point and Mach number distribution at $x=1.5$.

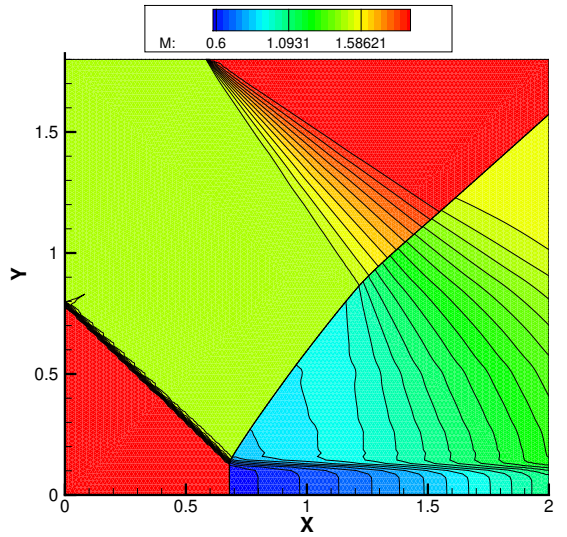

(a) $\mathrm{SF}$

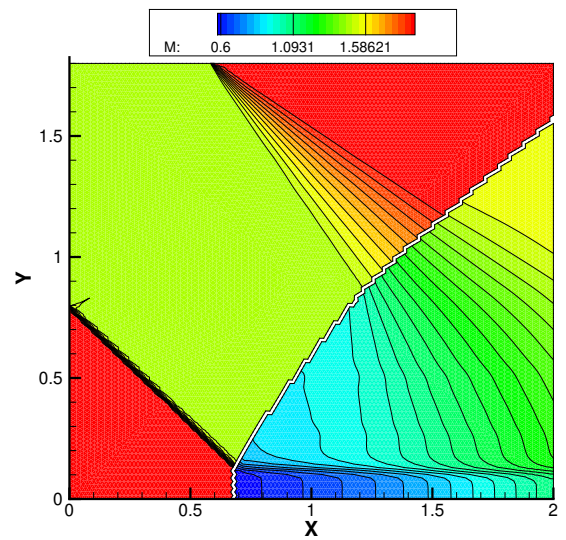

(b) eST

Figure 23: Steady Mach reflection: comparison between SF and eST in terms of density iso-contour lines.

of a circular cylinder. The resulting flow features a bow shock, interacting with the oblique shock, and giving rise to the well known type IV interaction, 


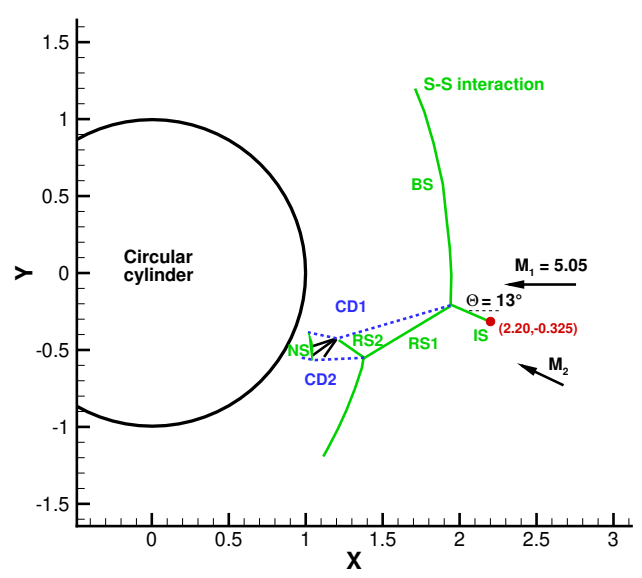

Figure 24: Sketch of the type IV shock-shock interaction problem.

which has been already studied in [15]. For clarity, we have drawn in Fig. 24 a sketch of this interaction. A first triple point (TP1) occurs where the oblique shock (IS) impinges on the bow shock giving rise to a reflected shock (RS1) and a contact discontinuity (CD1) that move towards the stagnation point. The contact discontinuity separates the supersonic stream which has been deflected by the oblique shock from the subsonic stream downstream of the bow shock (BS). The reflected shock coming from the first triple point re-joins the bow shock in a second triple point (TP2) where a new reflected shock (RS2) and contact discontinuity (CD2) arise. The two contact discontinuities bound a supersonic jet which is directed toward the body surface. Within the jet the second reflected shock interacts with the first contact discontinuity giving rise to an expansion wave. The flow concludes his path by being decelerated by a normal shock (NS) right in front of the body surface causing a higher density and pressure zone on the cylinder surface.

These kinds of interactions are very difficult to study because they require a very fine triangulation in order to properly describe what is going on within the flow-field. A Delaunay mesh containing 49660 triangles and 25231 nodes was used to compute the SC solution and also as background mesh for the hybrid computation in which eST was used to fit the entire bow-shock and the oblique shock RS1. Figures 25.a and 25.b show the differences between the two solutions in terms of density and Mach number iso-contours. The 


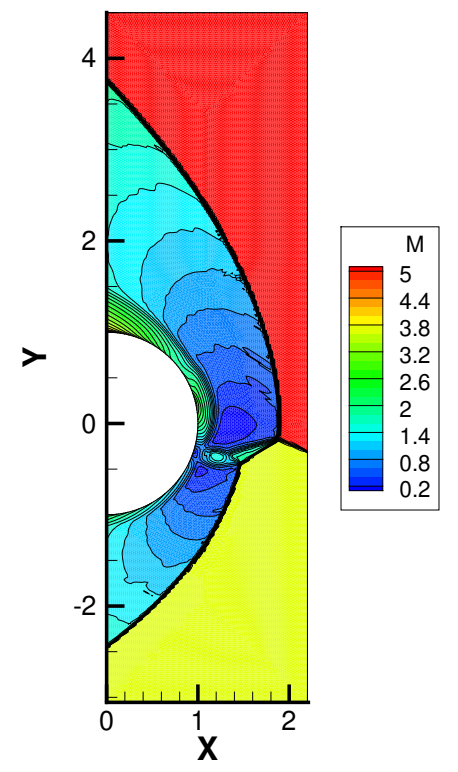

(a) $\mathrm{SC}$

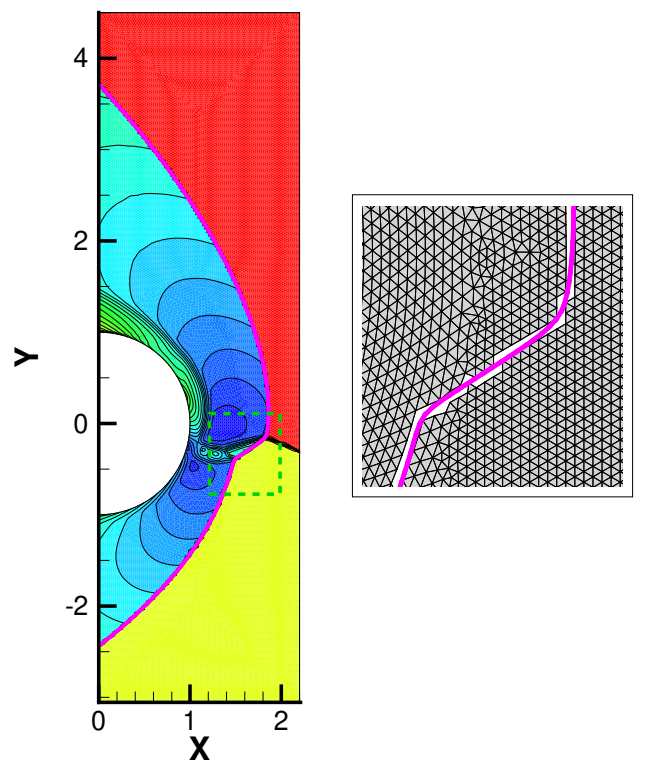

(b) eST

Figure 25: Type IV shock-shock interaction: Mach number iso-contours and a close-up on the shock-mesh.

pink bold line that appears in Fig. 25.b represents the fitted shock, better displayed in the close-up. As before, the use of eST allows to obtain a considerably smoother flow-field inside the shock layer.

\section{Conclusions and perspectives}

A novel technique to simulate flows with shock waves has been illustrated and tested on several applications on one-dimensional and two-dimensional unstructured grids. The proposed extrapolated shock tracking method borrows ideas from embedded boundary methods, combining them with a floating shock-fitting approach. The resulting technique has been proven to be able to provide genuinely second order results for flows with very strong shocks, without the complexity of the re-meshing phase of the previous fitting approaches. The method proposed has great potential in constructing 
generic shock-fitting/tracking strategies, with little dependence on the data structure of the underlying flow solver. As all shock/front tracking methods it has the enormous advantage of solving the exact jump conditions across the discontinuity, which makes these methods very competitive with any kind of adaptive capturing procedure, unless these conditions are embedded in the discretization, as done in some DG-based recent work [34, 35]. This however requires to set up a dedicated solver, while our approach has potential to be coupled with several different existing CFD codes.

Indeed one of the future challenges will be to compare its performance when coupled with different CFD solvers, not only unstructured cell-vertex, but also cell-centered and fully structured/Cartesian codes. Space for improvement of the method is clearly present with respect to its capability to handle explicitly interactions, improving the accuracy of the solution transfer to/from the shock, treating moving and complex three-dimensional discontinuities, and going beyond second order of accuracy.

\section{Appendix A. Gradient reconstruction}

The truncated Taylor series expansion (15) which is used to transfer the dependent variable $\mathbf{Z}$ between the surrogate boundaries and the shock-mesh relies on the availability of the gradient $\nabla \mathbf{Z}_{i}(\tilde{\mathbf{x}})$ in points, such as $A^{i}$ in Fig. 6 , and $B^{i}$ in Fig. 7 , which, respectively, belong to the surrogate boundary $\tilde{\Gamma}_{U}$ and $\tilde{\Gamma}_{D}$. As explained in Sect. 5.6, the calculation of the gradient in $A^{i}$ or $B^{i}$, by means of Eq. (16), requires the knowledge of the gradient in the grid-points of the surrogate boundaries.

Since the dependent variable $\mathbf{Z}$ is stored in the grid-points of the triangulation and varies linearly in space, $\nabla \mathbf{Z}$ is not readily available within the grid-points, but it has to be reconstructed there using the cell-wise constant gradient of the cells that surround a given grid-point, as sketched in Fig. A.26a. More precisely, the following area-weighted average is used ${ }^{3}$ :

$$
\nabla \mathbf{Z}_{i}=\frac{\sum_{i \ni T}\left(A_{T} \nabla \mathbf{Z}_{T}\right)}{\sum_{i \ni T} A_{T}}
$$

where the summation ranges over all the triangles that surround grid-point $i$ and $A_{T}$ denotes the triangle area. The cell-wise constant gradient $\nabla \mathbf{Z}_{T}$ that

\footnotetext{
${ }^{3}$ In this Appendix the notation $\mathbf{Z}_{i}$ collectively refers to the four components of $\mathbf{Z}$ in grid-point $i$, rather than to the $i^{\text {th }}$ component of $\mathbf{Z}$.
} 


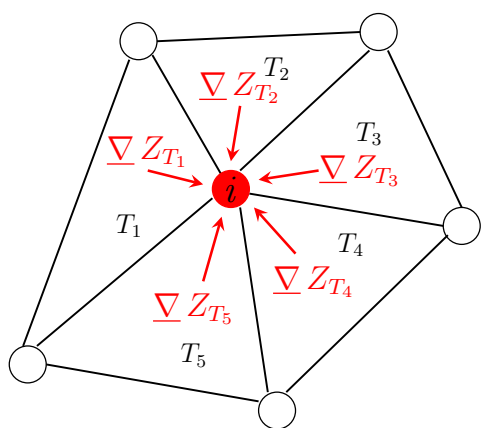

(a) The gradient in grid-point $i$ is computed by collecting fractions of the cell-wise constant gradients of all the cells that surround $i$.

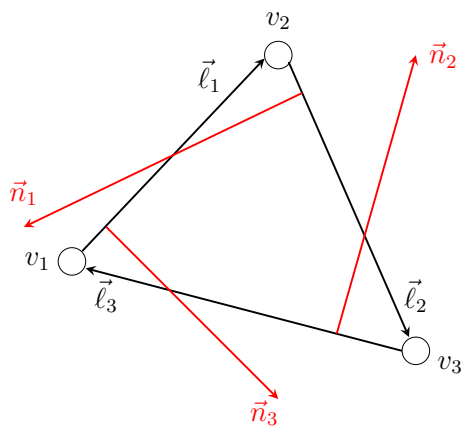

(b) Grid elements and vectors normal to the edges of the triangle.

Figure A.26: Reconstruction of the gradient.

appears in Eq. (A.1) can be easily computed using the values of $\mathbf{Z}$ within the vertices of triangle $T$ and the inward normals to the edges of the triangle, scaled by the edge length $\ell$ :

$$
\nabla \mathbf{Z}_{T}=\frac{\sum_{i=1,3}\left(\mathbf{Z}_{i} \mathbf{n}_{i}\right)}{2\left|A_{T}\right|}
$$

Figure A.26b clarifies the nomenclature used in Eq. (A.2).

The gradient reconstruction described so far applies to the two-dimensional case.

In the quasi-one-dimensional framework, see Fig. A.27 for a sketch of the 1D grid, the aforementioned approach boils down to the following one-sided finite-difference formula:

$$
\nabla \mathbf{Z}(\tilde{x})=\frac{\mathbf{Z}\left(x_{i+1}\right)-\mathbf{Z}\left(x_{i}\right)}{x_{i+1}-x_{i}}
$$

which approximates the gradient at the surrogate boundary, i.e. where $x=\tilde{x}$. The extrapolated value of $\mathbf{Z}$ at the discontinuity is obtained from:

$$
\mathbf{Z}(x)=\mathbf{Z}(\tilde{x})+\frac{\mathbf{Z}\left(x_{i+1}\right)-\mathbf{Z}\left(x_{i}\right)}{x_{i+1}-x_{i}}(x-\tilde{x})+o\left(\|x-\tilde{x}\|^{2}\right)
$$

where $\tilde{x}=x_{i}$ and $x=x_{s}$ are, respectively, the coordinates of the surrogate boundary and of the shock-point. 


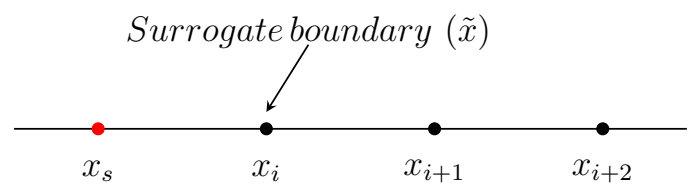

Figure A.27: Elements used to build the gradient on a one-dimensional grids

\section{Appendix B. Pseudo-temporal evolution and iterative convergence of the extrapolated Shock Tracking technique.}

In this Appendix we give further insight into the pseudo-temporal evolution of the flow-field to show how the eST algorithm, starting from a converged SC solution used as initial condition, leads to a steady, oscillation-free, shock-fitted result.

Figures B.28, B.29 and B.30 show a sequence of three frames that refer to different instances of the pseudo-temporal evolution of the solution for the three test-cases already described in Sects. 6.2, 6.4 and 6.5. In order to improve readability, the shock-mesh has not been plotted. It can be seen that the eST method requires a few hundred pseudo-time steps to get rid of the severe oscillations inherited by the SC calculation used to initialize the flow-field. Further iterations are required while the shock slows down, up to the point when its speed vanishes and it settles to its steady location. Convergence of the shock-mesh is monitored by computing, at each iteration, a mean shock velocity, averaged over all shock-points. Fig. B.31 shows the pseudo-temporal evolution of the mean shock velocity, plotted against the iteration counter, for all three test-cases. The solution is considered to be converged when this parameter experiences a notable drop, that might also be of several order of magnitude depending on the shock initial position, as the ones shown in Fig. B.31.

\section{References}

[1] D. Zaide, P. Roe, Shock capturing anomalies and the jump conditions in one dimension, in: 20th AIAA Computational Fluid Dynamics Conference, 2011, p. 3686 .

[2] J. Quirk, A contribution to the great Riemann solver debate, Interna- 


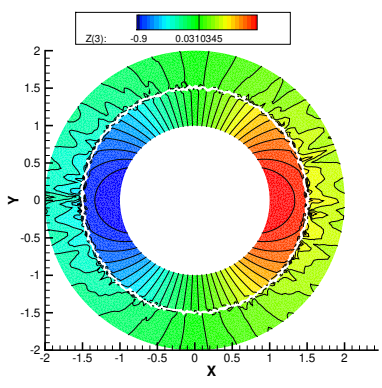

(a) Iteration 1

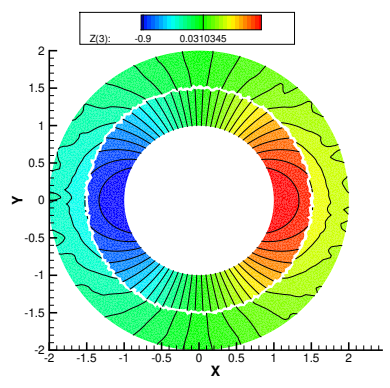

(b) Iteration 201

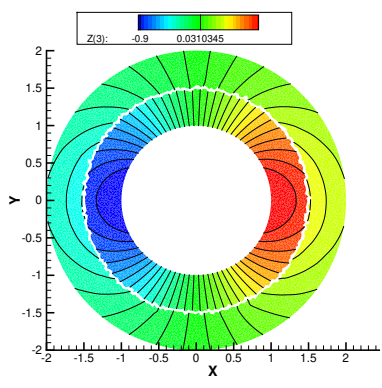

(c) Converged solution

Figure B.28: Planar, transonic source flow: pseudo-temporal evolution of the eST simulation starting from a SC solution (in terms of $\sqrt{\rho} u$ ).

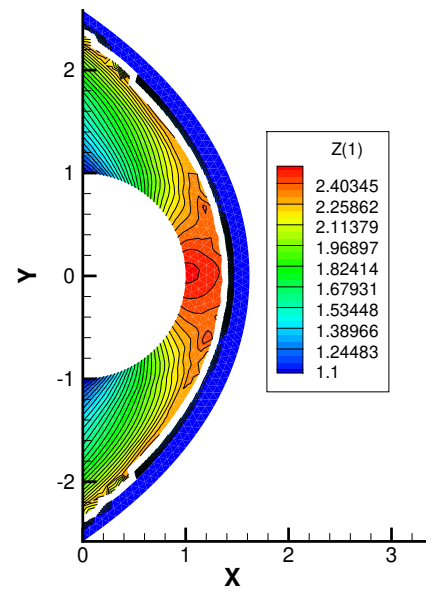

(a) Iteration 1

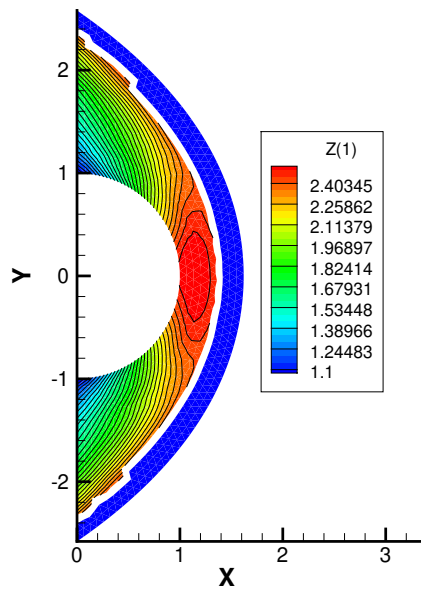

(b) Iteration 101

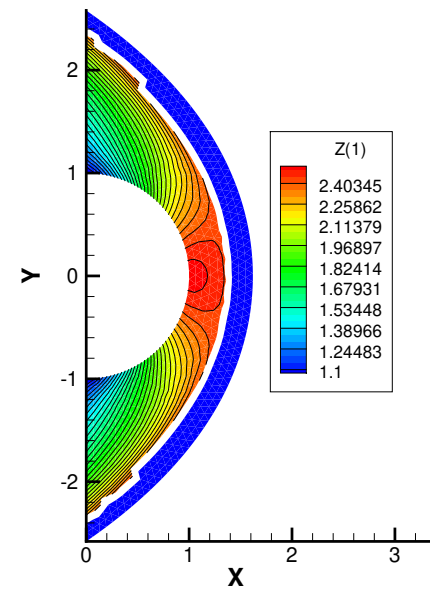

(c) Converged solution

Figure B.29: Hypersonic flow past a circular cylinder: pseudo-temporal evolution of the eST simulation starting from a SC solution (in terms of $\sqrt{\rho}$ ).

tional Journal for Numerical Methods in Fluids 18 (6) (1994) 555-574. doi:10.1002/fld.1650180603.

[3] G. Moretti, Three-dimensional, supersonic, steady flows with any number of embedded shocks, in: 12th Aerospace Sciences Meeting, 1974, p. 10. doi:10.2514/6.1974-10. 


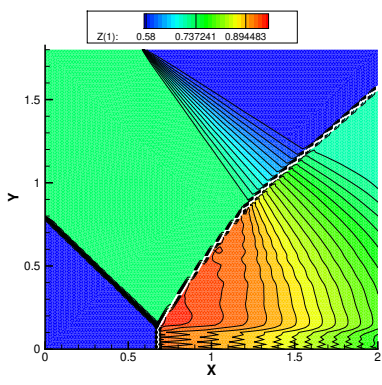

(a) Iteration 1

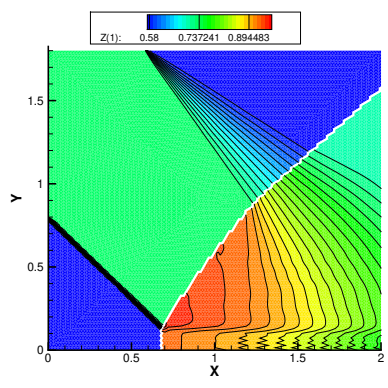

(b) Iteration 201

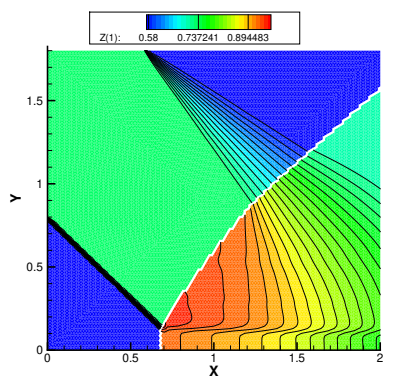

(c) Converged solution

Figure B.30: Steady Mach reflection: pseudo-temporal evolution of the eST simulation starting from a SC solution (in terms of $\sqrt{\rho}$ ).

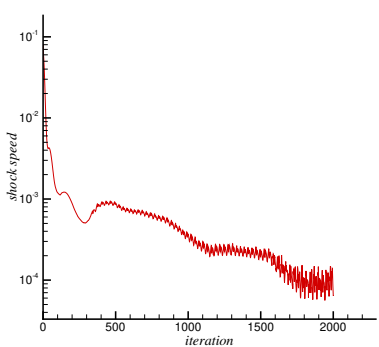

(a)

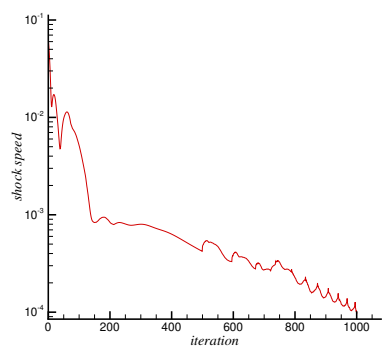

(b)

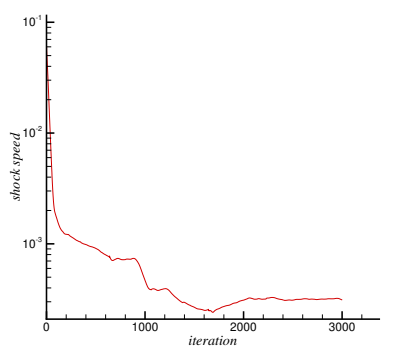

(c)

Figure B.31: Shock speed approaching steady state: (a) Planar source flow; (b) Blunt body problem; (c) Steady Mach reflection.

[4] R. Marsilio, G. Moretti, Shock-fitting method for two-dimensional inviscid, steady supersonic flows in ducts, Meccanica 24 (4) (1989) 216-222. doi:10.1007/BF01556453.

[5] F. Nasuti, M. Onofri, Analysis of unsteady supersonic viscous flows by a shock-fitting technique, AIAA journal 34 (7) (1996) 1428-1434. doi:10.2514/6.1995-2159.

[6] F. Nasuti, A multi-block shock-fitting technique to solve steady and unsteady compressible flows, in: Computational Fluid Dynamics 2002, Springer, 2003, pp. 217-222. 
[7] A. Prakash, N. Parsons, X. Wang, X. Zhong, High-order shock-fitting methods for direct numerical simulation of hypersonic flow with chemical and thermal nonequilibrium, Journal of Computational Physics 230 (23) (2011) 8474-8507. doi:10.1016/j.jcp.2011.08.001.

[8] R. Paciorri, A. Bonfiglioli, A shock-fitting technique for 2D unstructured grids, Computers \& Fluids 38 (3) (2009) 715-726. doi:10.1016/j.compfluid.2008.07.007.

[9] R. Paciorri, A. Bonfiglioli, Shock interaction computations on unstructured, two-dimensional grids using a shock-fitting technique, Journal of Computational Physics 230 (8) (2011) 3155-3177. doi:10.1016/j.jcp.2011.01.018.

[10] A. Bonfiglioli, M. Grottadaurea, R. Paciorri, F. Sabetta, An unstructured, three-dimensional, shock-fitting solver for hypersonic flows, Computers \& Fluids 73 (2013) 162-174. doi:10.1016/j.compfluid.2012.12.022.

[11] A. Bonfiglioli, R. Paciorri, L. Campoli, Unsteady shock-fitting for unstructured grids, International Journal for Numerical Methods in Fluids 81 (4) (2016) 245-261. doi:10.1002/fld.4183.

[12] L. Campoli, P. Quemar, A. Bonfiglioli, M. Ricchiuto, Shock-fitting and predictor-corrector explicit ale residual distribution, in: Shock Fitting, Springer, 2017, pp. 113-129. doi:10.1007/978-3-319-68427-7_5.

[13] M. S. Ivanov, A. Bonfiglioli, R. Paciorri, F. Sabetta, Computation of weak steady shock reflections by means of an unstructured shock-fitting solver, Shock Waves 20 (4) (2010) 271-284. doi:10.1007/s00193-0100266-y.

[14] D. Zou, C. Xu, H. Dong, J. Liu, A shock-fitting technique for cell-centered finite volume methods on unstructured dynamic meshes, Journal of Computational Physics 345 (2017) 866-882. doi:10.1016/j.jcp.2017.05.047.

[15] S. Chang, X. Bai, D. Zou, Z. Chen, J. Liu, An adaptive discontinuity fitting technique on unstructured dynamic grids, Shock Waves 29 (8) (2019) 1103-1115. doi:10.1007/s00193-019-00913-3. 
[16] C. S. Peskin, Flow patterns around heart valves: a numerical method, Journal of computational physics 10 (2) (1972) 252-271. doi:10.1016/0021-9991(72)90065-4.

[17] D. Boffi, L. Gastaldi, A finite element approach for the immersed boundary method, Computers \& Structures 81 (8-11) (2003) 491-501. doi:10.1016/S0045-7949(02)00404-2.

[18] R. Abgrall, H. Beaugendre, C. Dobrzynski, An immersed boundary method using unstructured anisotropic mesh adaptation combined with level-sets and penalization techniques, Journal of Computational Physics 257 (2014) 83-101. doi:10.1016/j.jcp.2013.08.052.

[19] L. Nouveau, H. Beaugendre, C. Dobrzynski, R. Abgrall, M. Ricchiuto, An adaptive, residual based, splitting approach for the penalized NavierStokes equations, Computer Methods in Applied Mechanics and Engineering 303 (2016) 208-230. doi:10.1016/j.cma.2016.01.009.

[20] A. Hansbo, P. Hansbo, An unfitted finite element method, based on Nitsche's method, for elliptic interface problems, Computer methods in applied mechanics and engineering 191 (47-48) (2002) 5537-5552. doi:10.1016/S0045-7825(02)00524-8.

[21] E. Burman, Ghost penalty, Comptes Rendus Mathematique 348 (21-22) (2010) 1217-1220. doi:10.1016/j.crma.2010.10.006.

[22] E. Burman, P. Hansbo, Fictitious domain methods using cut elements: Iii. a stabilized Nitsche method for Stokes' problem, ESAIM: Mathematical Modelling and Numerical Analysis 48 (3) (2014) 859-874. doi:10.1051/m2an/2013123.

[23] R. P. Fedkiw, T. Aslam, B. Merriman, S. Osher, A non-oscillatory eulerian approach to interfaces in multimaterial flows (the ghost fluid method), Journal of computational physics 152 (2) (1999) 457-492. doi:10.1006/jcph.1999.6236.

[24] C. Farhat, A. Rallu, S. Shankaran, A higher-order generalized ghost fluid method for the poor for the three-dimensional two-phase flow computation of underwater implosions, Journal of Computational Physics 227 (16) (2008) 7674-7700. doi:10.1016/j.jcp.2008.04.032. 
[25] A. Main, G. Scovazzi, The shifted boundary method for embedded domain computations. part I: Poisson and Stokes problems, Journal of Computational Physics 372 (2018) 972-995. doi:10.1016/j.jcp.2017.10.026.

[26] A. Main, G. Scovazzi, The shifted boundary method for embedded domain computations. part II: Linear advection-diffusion and incompressible Navier-Stokes equations, Journal of Computational Physics 372 (2018) 996-1026. doi:10.1016/j.jcp.2018.01.023.

[27] T. Song, A. Main, G. Scovazzi, M. Ricchiuto, The shifted boundary method for hyperbolic systems: Embedded domain computations of linear waves and shallow water flows, Journal of Computational Physics 369 (2018) 45-79. doi:10.1016/j.jcp.2018.04.052.

[28] D. She, R. Kaufman, H. Lim, J. Melvin, A. Hsu, J. Glimm, Front tracking methods, in: Handbook of Numerical Methods for Hyperbolic Problems, Vol. 17, Elsevier, 2016, pp. 383-402. doi:10.1016/bs.hna.2016.07.004.

[29] K. Li, N. M. Atallah, G. A. Main, G. Scovazzi, The shifted interface method: A flexible approach to embedded interface computations, International Journal for Numerical Methods in Engineering 121 (3) (2020) 492-518. doi:10.1002/nme.6231.

[30] R. Pepe, A. Bonfiglioli, A. DAngola, G. Colonna, R. Paciorri, An unstructured shock-fitting solver for hypersonic plasma flows in chemical non-equilibrium, Computer Physics Communications 196 (2015) 179193. doi:10.1016/j.cpc.2015.06.005.

[31] A. Lani, V. De Amicis, SF: An open source object-oriented platform for unstructured shock-fitting methods, in: M. Onofri, R. Paciorri (Eds.), Gino Moretti's Memoires, Fundamentals and Recent Evolution of his Shock-Fitting Technique, Springer International Publishing, 2017, pp. 127-153. doi:10.1007/978-3-319-68427-7_4.

[32] R. Paciorri, A. Bonfiglioli, Accurate detection of shock waves and shock interactions in two-dimensional shock-capturing solutions, Journal of Computational Physics $406 \quad$ (2020) 109196. doi:https://doi.org/10.1016/j.jcp.2019.109196. 
[33] L. Nouveau, M. Ricchiuto, G. Scovazzi, High-order gradients with the shifted boundary method: An embedded enriched mixed formulation for elliptic pdes, Journal of Computational Physics 398 (2019) 108898. doi:10.1016/j.jcp.2019.108898.

[34] M. Zahr, A. Shi, P.-O. Persson, Implicit shock tracking using an optimization-based high-order discontinuous galerkin method, Journal of Computational Physics 410 (2020) 109385. doi:10.1016/j.jcp.2020.109385.

[35] A. Corrigan, A. D. Kercher, D. A. Kessler, A moving discontinuous galerkin finite element method for flows with interfaces, International Journal for Numerical Methods in Fluids 89 (9) (2019) 362-406. doi:10.1002/fld.4697.

[36] A. Bonfiglioli, Fluctuation splitting schemes for the compressible and incompressible Euler and Navier-Stokes equations, International Journal of Computational Fluid Dynamics 14 (1) (2000) 21-39. doi:10.1080/10618560008940713.

[37] H. Deconinck, M. Ricchiuto, Residual Distribution Schemes: Foundations and Analysis, John Wiley \& Sons, Ltd, 2017, pp. 1-53. doi:10.1002/0470091355.ecm054.

[38] R. Abgrall, M. Ricchiuto, High-Order Methods for CFD, John Wiley \& Sons, Ltd, 2017, pp. 1-54. doi:10.1002/9781119176817.ecm2112.

[39] A. Bonfiglioli, R. Paciorri, A mass-matrix formulation of unsteady fluctuation splitting schemes consistent with Roe's parameter vector, International Journal of Computational Fluid Dynamics 27 (4-5) (2013) 210-227. doi:10.1080/10618562.2013.813491.

[40] P. L. Roe, Approximate riemann solvers, parameter vectors, and difference schemes, Journal of computational physics 43 (2) (1981) 357-372. doi:10.1006/jcph.1997.5705.

[41] D. Bonhaus, A higher order accurate finite element method for viscous compressible flows, Ph.D. thesis, Virginia Polytechnic Institute and State University (1998). 
[42] A. Bonfiglioli, R. Paciorri, Convergence analysis of shock-capturing and shock-fitting solutions on unstructured grids, AIAA journal 52 (7) (2014) 1404-1416. doi:10.2514/1.J052567.

[43] M. S. Campobasso, M. H. Baba-Ahmadi, Ad-Hoc Boundary Conditions for CFD Analyses of Turbomachinery Problems With Strong Flow Gradients at Farfield Boundaries, Journal of Turbomachinery 133 (4). doi:10.1115/1.4002985.

[44] J. R. Shewchuk, Triangle: Engineering a 2d quality mesh generator and delaunay triangulator, in: Workshop on Applied Computational Geometry, 1996, pp. 203-222. doi:10.1007/BFb0014497.

[45] J. Shewchuk, Triangle mesh generator, Available at https://www.cs.cmu.edu/ quake/triangle.html.

[46] M. Grottadaurea, R. Paciorri, A. Bonfiglioli, F. Sabetta, M. Onofri, D. Bianchi, Numerical simulation of hypersonic flows past threedimensional blunt bodies through an unstructured shock-fitting solver., in: International Space Planes and Hypersonic Systems and Technologies Conferences, American Institute of Aeronautics and Astronautics, 2011, pp. -. doi:10.2514/6.2011-2288.

[47] F. Alauzet, A. Loseille, G. Olivier, Time-accurate multiscale anisotropic mesh adaptation for unsteady flows in cfd, Journal of Computational Physics 373 (2018) 28 - 63. doi:https://doi.org/10.1016/j.jcp.2018.06.043.

[48] J.-D. Müller, P. L. Roe, H. Deconinck, A frontal approach for internal node generation in delaunay triangulations, International Journal for Numerical Methods in Fluids 17 (3) (1993) 241-255. doi:10.1002/fld.1650170305.

[49] J. Müller, Delaundo mesh generator, Available at http://www.ae.metu.edu.tr/tuncer/ae546/prj/delaundo/.

[50] A. Lyubimov, V. Rusanov, Gas Flows Past Blunt Bodies, Part II: Tables of the Gasdynamic Functions, NASA TT F-715.

[51] N. Duquesne, T. Alziary de Roquefort, Numerical investigation of a three-dimensional turbulent shock/shock interaction, in: 36th 
AIAA Aerospace Sciences Meeting and Exhibit, 1998, p. 774. doi:10.2514/6.1998-774. 